\title{
A new analytic ram pressure profile for satellite galaxies
}

\author{
Cristian A. Vega-Martínez, ${ }^{1,2 \star}$ Facundo A. Gómez, ${ }^{1,2}$ Sofía A. Cora, ${ }^{3,4}$ Tomás Hough ${ }^{3,4}$ \\ ${ }^{1}$ Instituto de Investigación Multidisciplinar en Ciencia y Tecnología, Universidad de La Serena, Raúl Bitrán 1305, La Serena, Chile \\ 2 Departamento de Astronomía, Universidad de La Serena, Av. Juan Cisternas 1200 Norte, La Serena, Chile \\ ${ }^{3}$ Instituto de Astrofísica de La Plata (CCT La Plata, CONICET, UNLP), Paseo del Bosque s/n, La Plata, Argentina \\ ${ }^{4}$ Facultad de Ciencias Astronómicas y Geofísicas, Universidad Nacional de La Plata, Paseo del Bosque s/n, La Plata, Argentina
}

Accepted XXX. Received YYY; in original form ZZZ

\begin{abstract}
We present a new analytic fitting profile to model the ram pressure exerted over satellite galaxies on different environments and epochs. The profile is built using the information of the gas particle distribution in hydrodynamical simulations of groups and clusters of galaxies to measure the ram pressure directly. We show that predictions obtained by a previously introduced $\beta$-profile model can not consistently reproduce the dependence of the ram pressure on halocentric distance and redshift for a given halo mass. It features a systematic underestimation of the predicted ram pressure at high redshifts $(z>1.5)$, which increases towards the central regions of the haloes and it is independent of halo mass, reaching differences larger than two decades for satellites at $r<0.4 R_{\text {vir }}$. This behaviour reverses as redshift decreases, featuring an increasing over-estimation with halocentric distance at $z=0$. As an alternative, we introduce a new universal analytic model for the profiles which can recover the ram pressure dependence on halo mass, halocentric distance and redshift. We analyse the impact of our new profile on galaxy properties by applying a semi-analytic model of galaxy formation and evolution on top of the simulations. We show that galaxies experiencing large amounts of cumulative ram pressure stripping typically have low stellar masses $\left(M_{\star} \leq 10^{9.5} \mathrm{M}_{\odot}\right)$. Besides, their specific star formation histories depend on the ram pressure modelling applied, particularly at high redshifts $(z>1.5)$.
\end{abstract}

Key words: galaxies: general - galaxies: interactions - galaxies: evolution-galaxies: clusters: general - methods: numerical

\section{INTRODUCTION}

During the last decades, observations of the galaxy population inhabiting different environments have shown a clear bimodal distribution in several galaxy properties including colours, morphology, stellar ages and star formation rates, among others (e.g. Kauffmann et al. 2003; Baldry et al. 2004; Cassata et al. 2008; Thomas et al. 2010; Peng et al. 2010; Wetzel et al. 2012; Taylor et al. 2015). This suggests a division between galaxies dominated by recently formed stellar populations and galaxies with old stellar content. A critical difference between these populations is the depletion of the global star formation activity. This state, also referred to as galaxy quenching, is generally defined when the specific star formation rate (sSFR) of a galaxy, i.e. the rate of stars formed divided by its stellar mass, decreases below a certain value, being $10^{-11} \mathrm{yr}^{-1}$ the usual threshold for galaxies at $z=0$ (Weinmann et al. 2010; De Lucia et al. 2012; Wetzel et al. 2013).

The contribution to the star formation quenching from the different physical processes driving galaxy evolution is still a topic

^ E-mail: cristian.vegam@userena.cl of debate (see Somerville \& Davé 2015, for a review on physical models on galaxy formation). Two different types of processes have been invoked in the suppression of the star formation: massand environmental- quenching (Peng et al. 2010). Comparisons between the properties of populations of star-forming (active) and quiescent (passive) galaxies have shown that, up to $z \sim 1$, it is possible to identify the main mechanism driving them to the quenching state (e.g. Baldry et al. 2006; Peng et al. 2010; Muzzin et al. 2012; Kovač et al. 2014; Guglielmo et al. 2015; van der Burg et al. 2018, 2020). This, however, does not necessarily mean that they are physically unrelated. At higher redshifts, the picture is more intriguing. The median star formation rate (SFR) of galaxies and the quenched fraction (i.e. the ratio between the number of quenched galaxies and the total number of galaxies, including quenched and star-forming ones) are observed to be independent of the environment (e.g. Darvish et al. 2016). However, it has also been argued that the environment can have an impact on galaxy quenching even up to $z \sim 1.6$ in systems associated to the largest overdensities (Nantais et al. 2016, 2017).

Environmental-quenching includes all physical mechanisms affecting galaxies according to the environment where they evolve 
(see Boselli \& Gavazzi 2006, for a comprehensive description of these processes). At the same stellar mass, the measured fraction of quenched galaxies is larger for satellite galaxies than for centrals (e.g Peng et al. 2012), and the differences increase strongly with main host halo mass (e.g Wetzel et al. 2012), and towards the halo centre (e.g. Haines et al. 2015). There are indications that the influence of the environment is stronger for low-mass satellite galaxies $\left(M_{\star} \lesssim 10^{10} \mathrm{M}_{\odot}\right)$ rather than for higher mass ones (Haines et al. 2006; Bamford et al. 2009; Peng et al. 2010; Roberts et al. 2019), and this has been supported by theoretical analysis (e.g. De Lucia et al. 2012; Cora et al. 2018; Xie et al. 2020). Besides, environmental quenching is more evident when analysing the largest over-densities, where galaxy clusters are located $\left(M_{\text {halo }} \approx 10^{15} \mathrm{M}_{\odot}\right.$ ). Analysis of dense regions in the local Universe have shown higher fractions of quenched galaxies (e.g. van den Bosch et al. 2008; Gavazzi et al. 2010), being the sSFR of the satellite galaxies significantly smaller in clusters than in lower density regions (e.g. Haines et al. 2013), and this behaviour is also present in high redshift clusters (e.g. van der Burg et al. 2018, 2020).

The suppression of the gas accretion onto galaxy discs (usually referred to as starvation, Larson et al. 1980) and the loss of gas from the galaxy discs due to the interaction with the medium through ram pressure stripping (RPS, Gunn \& Gott 1972) have been the two processes most commonly associated to the quenching of satellite galaxies in galaxy clusters (Quilis et al. 2000; Wetzel et al. 2013; Muzzin et al. 2014; Peng et al. 2015; Jaffé et al. 2015), and also in the Local Group (e.g. Fillingham et al. 2015; Wetzel et al. 2015). A critical difference between the effect of each process is the quenching timescale inferred from the galaxy properties. For starvation, once the feeding of gas to the satellite halts, the star formation of the galaxy can be suppressed in $\gtrsim 4$ Gyr (e.g. Peng et al. 2015), whereas RPS of the gas disc could quench the galaxy in much shorter timescales $\lesssim 1$ Gyr (e.g. Quilis et al. 2000; Roediger \& Hensler 2005; Steinhauser et al. 2016). Furthermore, observations of galaxy groups have shown that the quenching of dwarf galaxies $\left(M_{\star} \lesssim 10^{8} \mathrm{M}_{\odot}\right)$ is mainly driven by ram pressure, whereas starvation dominates quenching of galaxies with $M_{\star} \gtrsim 10^{8} \mathrm{M}_{\odot}$ (Fillingham et al. 2015; Wetzel et al. 2015). Nonetheless, recent observations indicate that environment may have a negligible role in the quenching of ultra faint galaxies observed in the Local Group (Rodriguez Wimberly et al. 2019). The differentiation between these processes becomes more complicated when we consider that ram pressure can also strip part of the hot halo gas of the galaxy, without necessarily acting over the disc, leading to a starvation scenario (Bekki et al. 2002; Steinhauser et al. 2016). Furthermore, another environmental process that can contribute to the star formation quenching of satellite galaxies is the stripping of gas and stars via tidal interactions (Merritt 1983). Tidal stripping is, however, considered as a secondary effect in comparison with RPS (e.g. McCarthy et al. 2008; Font et al. 2008; Bahé \& McCarthy 2015), agreeing with inferences from observational results (e.g. Boselli et al. 2016).

Due to the hierarchical growing of the dark matter haloes (White \& Rees 1978; Davis et al. 1985), a large fraction of the satellites of $z=0$ massive clusters is predicted to have been accreted as part of less-massive groups (McGee et al. 2009), thus galaxy quenching can start in these groups in a pre-processing stage before falling into larger systems (e.g. De Lucia et al. 2012; Jaffé et al. 2015; Pallero et al. 2019, 2020), Moreover, it has been shown that satellites and central galaxies can be pre-processed at several virial radius from a cluster centre (e.g. Bahé et al. 2012; Zinger et al.
2018; Ayromlou et al. 2021). Recent analysis have shown a strong dependence of the quenching fraction on the intra-cluster medium (ICM) density, featuring a specific threshold that determines a sharp increase in the quenching efficiency (Roberts et al. 2019; Pallero et al. 2020), raising the connection between the different environments and the ram pressure exerted over the satellites residing in them, and favouring the importance of RPS as a crucial mechanism to understand the general galaxy quenching process.

The process of gas stripping from galaxies moving through dense environments like galaxy clusters has been explored using numerical simulations since the early studies of Lea \& De Young (1976); Gisler (1976); Toyama \& Ikeuchi (1980); Takeda et al. (1984). These studies calculated axially symmetric individual stripping scenarios with two-dimensional hydrodynamical codes. The first detailed three-dimensional, time-dependent hydrodynamical calculation of an elliptical galaxy orbiting in a gas-rich cluster was performed by Toniazzo \& Schindler (2001). They showed that stripping is less efficient than previously reported. In addition, the first three-dimensional hydrodynamical simulations of disc galaxies experiencing RPS on a cluster-like environment was presented by Roediger \& Brüggen (2007), showing that the mass loss history can not be accurately described by the Gunn \& Gott (1972) analytical estimate. Different techniques have been applied to study the stripping process acting on satellite galaxies within groups and clusters with hydrodynamical simulations. Among these techniques, we found simulations of isolated galaxies exposed to wind tunnel-like mediums to mimic their moving across dense environments (e.g. Roediger \& Hensler 2005; Roediger et al. 2006; Roediger \& Brüggen 2006; Kronberger et al. 2008; Bekki 2009; Tonnesen \& Bryan 2010, 2012; Ruszkowski et al. 2014), galaxies following orbits throughout galaxy clusters (e.g. Vollmer et al. 2001; Roediger \& Brüggen 2007, 2008; Jáchym et al. 2007; Tonnesen \& Bryan 2008; Heß \& Springel 2012; Vijayaraghavan \& Ricker 2013), and more realistic setups of populations of satellites being evolved in clusters (e.g. Vijayaraghavan \& Ricker 2015; Jung et al. 2018). These theoretical analysis have allowed to characterise the stripping process of galaxy hot gas haloes (e.g McCarthy et al. 2008; Vijayaraghavan \& Ricker 2015), the mass loss from the galaxy discs (e.g. Roediger \& Brüggen 2007, 2006; Bekki 2009, 2014; Ruggiero \& Lima Neto 2017), and even the formation and morphological evolution of the wakes and tails of stripped mass from discs (e.g. Roediger \& Brüggen 2008; Tonnesen \& Bryan 2010; Roediger et al. 2014; Ruszkowski et al. 2014). It has been shown that the external pressure of the ICM can produce an enhancement of the star formation in galaxy discs (e.g. Kronberger et al. 2008; Roediger et al. 2014; Bekki 2014) or in bulges (e.g. Tonnesen \& Bryan 2012). Moreover, the effect of the inclination angles of galaxy discs with respect to their orbital directions across different environments has been studied (e.g. Roediger \& Brüggen 2006; Bekki 2014), and it has also been shown that RPS can have an important effect on the galaxy properties on galaxy groups (e.g. Kawata 2008; Bekki 2009). Nonetheless, discrepancies on the stripping rate depending on the numerical technique applied to solve the hydrodynamical interactions in the gas modelling have been reported (Heß \& Springel 2012). Therefore, different strategies to model this environmental effect must be considered.

The semi-analytical models (SAMs) of galaxy formation and evolution are another useful technique broadly applied to study the processes driving the evolution of the galaxy properties in a cosmological context. In the last years, the use of these models has allowed to make important contributions to our understanding of the impact 
of RPS on galaxy properties. Recent SAMs with updated treatments for RPS on satellite galaxies, including gradual stripping of the hot gas and mass loss from discs (Stevens \& Brown 2017; Cora et al. 2018; Xie et al. 2020; Ayromlou et al. 2021), have shown that RPS acting on both components is an important ingredient to consistently predict the quenched fraction of satellites in different environments. In addition, Tecce et al. (2010) introduced a hybrid technique to model RPS in SAMs when they are applied on non-radiative hydrodynamical simulations of galaxy clusters. This treatment separates the modelling of the exerted pressure on a satellite from the mass loss due to the stripping, where the former is measured directly from the gas particle distribution of the simulation and the latter is modelled analytically inside the SAM. In this way, the uncertainties regarding to the modelling of the interaction between the gas phases (i.e. the stripping of the gas from the different galaxy components) can be explored analytically. With this approach they also showed that the assumption of an analytical density profile to describe the ICM give rise to an overestimation of the RPS at $z>0.5$. Subsequently, Tecce et al. (2011) introduced a fitted analytic profile to model the ram pressure on different environments, from measurements of the pressure in the same set of adiabatic hydrodynamical simulations of galaxy clusters. On the other hand, Ayromlou et al. (2019) introduced the Local Background Environment (LBE) technique to approximate ram pressure from dark matter-only $\mathrm{N}$-body simulations, based on a detailed analysis of the particle distribution of the simulation. Following these type of approaches, this work is focused on the ram pressure modelling using analytical estimations obtained from numerical simulations. The next subsection describes the modelling of ram pressure we consider in our analysis.

\subsection{Ram pressure estimation}

The ram pressure (RP) is the result of the interaction between the galaxies and the medium surrounding them, across which they are moving. In case of satellite galaxies, the RP is exerted by the ICM and is determined by the density of the medium in the satellite position and its orbital evolution. Specifically, it is defined by

$P_{\text {ram }} \equiv \rho_{\text {ICM }} v^{2}$,

where $\rho_{\text {ICM }}$ is the ICM density in the host halo and $v$ is the relative velocity between the satellite and the medium. Both quantities depend on the galactocentric distance of the satellite. Therefore, this strong dependence on satellite galactocentric distance must be accounted for when estimating the amount of stripped gas mass from the galaxy. When the gravitational restoring force of the galaxy is smaller than the RP, then the diffuse inter-stellar medium can be stripped from the satellite (Gunn \& Gott 1972; Abadi et al. 1999; Quilis et al. 2000; Vollmer et al. 2001; Jaffé et al. 2015). According to this definition, the estimation of the effective RP exerted over a particular observed galaxy requires to define a model of the host halo gas density profile, and to estimate the orbital galaxy speed relative to the host (e.g. Rasmussen et al. 2008; Jaffé et al. 2015, 2018; Roberts et al. 2019). When analysing numerical simulations, on the other hand, the estimation depends on the type of calculation considered.

In this work, we use adiabatic hydrodynamical simulations to analytically model the RP exerted on satellite galaxies inhabiting different environments. We use the properties of the gas particles considered in this type of simulations to directly measure the density of the ICM at the precise positions where the galaxies are located by applying the Tecce et al. (2010) technique, which allows to obtain smooth ICM density profiles in agreement with X-ray observations of galaxy clusters (as is shown in Tecce et al. 2010, figure 1). Thereby, these calculations allow to study the evolution of the RP across cosmic time, to measure its dependence with host halo mass, and to define analytic profiles to model it, as done by Tecce et al. (2011) (hereafter T11). These analytic profiles can be considered to consistently model the fraction of mass stripped from satellites by RP over their evolution with semi-analytic models of galaxy formation (e.g. Cora et al. 2018). SAMs are based on a set of analytic prescriptions to account for the main physical processes driving galaxy formation and thus can not self-consistently follow the spatial distribution of gas in the galaxies. Such models are usually coupled to dark matter halo properties and merger trees obtained from cosmological simulations, or created from a (extended) PressSchechter formalism (Press \& Schechter 1974; Bond et al. 1991; Lacey \& Cole 1993).

As we have already mentioned, there is increasing evidence indicating that RPS is a key process in galaxy evolution. Hence, a detailed, accurate and simple modelling of this environmental effect is becoming highly demanded. Accordingly, in this work we revisit the predictions of the analytic RP profile presented by T11, measured from cosmological hydrodynamical simulations. We show that it features important systematic differences between its predictions for the RP and the expected values measured in the simulations considered in its original calculation. Moreover, we go further introducing a new analytic profile able to overcome those differences in the predictions, achieving higher accuracy in the modelling. This paper is organised as follows. In Section 2, we describe the original T11 profile, detailing the simulations used in its estimation and we analyse the problems found in its predictions. In Section 3, we introduce the new analytic profile based on the same simulations and show the improvements regarding the predictions. In Section 4, we evaluate the impact of the usage of these profiles in the galaxy properties by the application of a SAM. Finally, in Section 5, we summarise our analysis highlighting the main results.

\section{T11 RAM PRESSURE PROFILE}

In T11, a set of N-body/Smooth Particle Hydrodynamical (SPH) adiabatic resimulations of clusters of galaxies were used to measure the RP exerted on galaxies and thus to estimate RP profiles as a function of the halocentric distances, redshifts and the host halo virial masses. The resulting shapes of the RP profiles within these haloes were characterised by a $\beta$-profile (Tecce et al. 2010), and a numerical fit of its parameters were provided as a main result. In the following, we describe the simulations used in this work (also considered by T11) and present an overview and a subsequent analysis of the T11 profile.

\subsection{The simulations}

As in T11, we use the set of non-radiative N-body/SPH resimulations of galaxy clusters described in Dolag et al. (2005, 2009), which were calculated following the entropy-conserving formulation of SPH (Springel \& Hernquist 2002) and considering the usual parametrization of artificial viscosity (tagged as ovisc in Tecce et al. 2010). These correspond to resimulations of selected regions from a $\sim 685 \mathrm{Mpc}$ side-length volume, characterised by a $\Lambda \mathrm{CDM}$ cosmology with $\Omega_{\mathrm{m}}=0.3, \Omega_{\Lambda}=0.7$, a Hubble constant $H_{0}=70 \mathrm{~km} \mathrm{~s}^{-1} \mathrm{Mpc}^{-1}$ and a power spectrum with normalization $\sigma_{8}=0.9$. The resimulations consider a universal baryonic density $\Omega_{\mathrm{b}}=0.039$, with a dark matter particle mass $1.13 \times 10^{9} h^{-1} \mathrm{M}_{\odot}$ and 
gas particle mass $1.69 \times 10^{8} h^{-1} \mathrm{M}_{\odot}$ (for more details about these simulations we refer the reader to Dolag et al. 2009). The identification of the dark matter haloes and the construction of their corresponding merger trees were done following Springel et al. (2001). Each system of dark matter haloes is characterised by the identification of a main host halo (i.e. the largest overdensity found over the background), which is detected through a friends-of-friends (FOF) technique (Davis et al. 1985). The centre of each halo was defined according to the position of its most bound dark matter particle. Properties like the virial mass and radius of the main host haloes were obtained from the resulting density profiles given by the distribution of the dark matter particles at each redshift. Assuming a spherical-overdensity criteria (Gunn \& Gott 1972), we define the virial mass $M_{200}$ of each main host halo as the mass contained in a sphere of radius $R_{200}$ at which the density equate a $\Delta$ factor of the critical density of the Universe. With a constant value of $\Delta=200$, the virial mass results

$M_{200}\left(<R_{200}\right)=200 \rho_{\mathrm{c}} \frac{4 \pi}{3} R_{200}^{3}$,

where $\rho_{\mathrm{c}}$ is the critical density of the Universe. Then, all the haloes lying within each main host (also referred to as subhaloes) were identified using SubFind (Springel et al. 2001), which defines subhalo particle members based on a binding energy criteria. Excluding the main subhalo, corresponding to the host of the central galaxy of each system, all the other resolved subhaloes are considered as satellites of the main host. Following these definitions, throughout this work, we use the term halo to refer indistinctly to any dark matter overdensity, independent if it is detected over the background density or inside another halo. We specify if we refer to a main host or a subhalo when needed.

The resimulations considered here are three regions centred in large overdensities corresponding to massive galaxy clusters with $M_{200} \sim 10^{15} h^{-1} \mathrm{M}_{\odot}$ (originally labelled g1, g8 and g51 in Dolag et al. 2005, 2009), and five regions corresponding to lowmass galaxy clusters with $M_{200} \sim 10^{14} h^{-1} \mathrm{M}_{\odot}$ (labelled g676, g914, g1542, g3344 and g6212). These high-resolution regions also include a set of resolved smaller clusters and groups in the surroundings of the central clusters. From those, we select the main host haloes that are free of particles with lower mass resolution, to create a sample of non-contaminated systems to be also considered in our analysis. Thereby, the analysed sample includes 759 systems in the mass range $11<\log \left(M_{200} h^{-1}\left[\mathrm{M}_{\odot}\right]\right)<14$, and 8 galaxy clusters with $\log \left(M_{200} h^{-1}\left[\mathrm{M}_{\odot}\right]\right)>14$.

\subsection{Ram pressure profile model}

The analytic RP profile introduced by T11 was obtained from the simulations described above, using the positions and velocities of the satellites to trace the RP experienced by them at different galactocentric distances, epochs, and ranges of main host halo masses. Additionally, they included the SAM of galaxy formation and evolution, SAG (Cora 2006; Lagos et al. 2008; Tecce et al. 2010), only to extend the sample of RP profile tracing points by incorporating the orphan satellite galaxies, which are followed by the semi-analytic modelling. These orphans are galaxies that have been hosted by haloes which are no longer detected by the halo finder because they have either merged with the main host halo or their masses decreased below the resolution limit of the simulation. Therefore, the positions and velocities of these satellites are defined according to the properties of the most bound particle of the halo to which each galaxy last belonged (see Tecce et al. 2010).
As was shown in T11, the efficiency of RP increases with the mass of the main host halo and with decreasing redshift. The best-fitting profile was found to be a full $\beta$-profile, commonly used for characterising the ICM around massive galaxies (e.g. Churazov et al. 2008). In this case, the profile is defined by

$P_{\text {ram }}(M, z)=P_{0}(M, z)\left[1+\left(\frac{r}{r_{\mathrm{s}}(M, z)}\right)^{2}\right]^{-\frac{3}{2} \beta(M, z)}$,

where the central value $P_{0}$, the characteristic radius $r_{\mathrm{s}}$ and the exponent $\beta$ are free parameters which depend on both the host halo mass and the redshift. In T11, these parameters were fitted for different samples, covering a set of bins in main host halo masses, and for each simulation snapshot in the redshift range $0 \leq z \leq 3$. To obtain the dependence of these parameters on the redshift, a linear relation in terms of the expansion factor of the Universe, $a$, was proposed according to

$$
\begin{aligned}
\log \left(\frac{P_{0}}{10^{-12} h^{2} \mathrm{dyn} \mathrm{cm}^{-2}}\right) & =A_{P}+B_{P}(a-0.25), \\
\frac{r_{\mathrm{s}}}{R_{200}} & =A_{r}+B_{r}(a-0.25), \\
\beta & =A_{\beta}+B_{\beta}(a-0.25),
\end{aligned}
$$

where all the coefficients $A_{i}$ and $B_{i}$ with $i=P, r$, or $\beta$ depend in principle on the main host virial mass. Additionally, a linear model following the expression $a+b\left(\log M_{200}-12\right)$ was proposed to parametrize the behaviour of each of the $A_{i}$ and $B_{i}$ coefficients, doubling in this way the total number of free parameters involved in the fits. The numerical result of these twelve coefficients are given by the equations (6a) to (6f) in T11. The resulting error associated to each one of them was variable, reaching a maximum value of 25 per cent in a couple of cases. It was also found that both $A_{\beta}$ and $B_{\beta}$ were not dependent on the main host halo masses. Consequently the $\beta$ parameter was expressed only as a function of the redshift. Thereby, all the RP profiles in the analysed redshift range were expressed in terms of ten numerical constants which define the shape of the profiles according to time and host halo mass for each satellite galaxy inhabiting a main host dark matter halo.

\subsection{Accuracy of the model}

Now we proceed to evaluate the reliability of the T11 RP profile model in predicting the effective RP exerted on satellite galaxies. To achieve a complete consistency between our analysis and their analytic RP profiles, the same set of hydrodynamical simulations are considered throughout our work. We extract all the positions of the satellite haloes belonging to main host haloes detected in the complete resimulated regions described in Section 2.1, excluding only the main haloes contaminated with boundary particles. This considerably extend the number of inspected main host in comparison with T11, particularly in the low-mass regime. Furthermore, this comparison is restricted to only use the positions of the satellite haloes identified by the halo finder, excluding all the extra tracers considered in T11 derived from the SAM (i.e. orphan satellites). Although this restriction reduces the number of points to be used to define the profiles, it also limits the analysis only to the resimulations and their (sub)haloes. We can therefore avoid the usage of a SAM in this calculation.

At each satellite position, we measure the effective RP obtained from the simulation, $P_{\text {ram }}^{\text {sim }}$, by following the method described in Tecce et al. (2010). Here, the ambient density and relative velocity with respect to the satellite are measured using the gas particles 
located in a sphere centred at the position of the satellite, excluding those associated to the subhalo. Specifically, we remove those neighbouring gas particles lying within 2.5 times the radius of the subhalo (as given by SuBFIND) having densities larger than twice the median gas density calculated from selected set of particles. This filtering procedure is repeated in an iterative process until the median density converges, thus recovering a smooth ICM without significantly affecting the median density profile. With these estimations, the equation (1) is directly applied. Besides, the predicted value of RP according to the T11 model, $P_{\text {ram }}^{\text {fit }}$, is also estimated at each satellite position.

The comparison between $P_{\mathrm{ram}}^{\mathrm{sim}}$ and $P_{\mathrm{ram}}^{\mathrm{fit}}$ is shown in Fig. 1 for three different redshifts, $z=0,1.5$ and 3, in different panels. Each one of the samples is separated according to the instantaneous main host halo mass, and they are indicated with different line styles. The mean ratios between the predicted and the measured values of the RP felt by the satellites are shown versus the corresponding relative radial distances to the centres of their main host haloes, $r / R_{200}$. As a reference, one standard deviation around the mean is also included in the figure through closed rectangles for two selected radial distances in each case: at $r / R_{200}=0.15$ for the most massive haloes found at each redshift, and at $r / R_{200}=0.85$ for the least massive ones. This allows us to compare more precisely the halo masses or redshifts at which the difference between the model and the measurements become more significant. As it can be appreciated from the figure, the T11 model overestimates the predicted RP at $z=0$ for more than 0.5 decades in most of the analysed main host halo masses, except for the most massive ones $\left(\log \left(M_{200}\left[h^{-1} \mathrm{M}_{\odot}\right]\right)>15\right)$ where the $P_{\text {ram }}^{\text {fit }} / P_{\text {ram }}^{\text {sim }}$ ratio monotonically decreases towards the centre of the hosts. Here, the profile underestimates the RP for satellites within distances $\leq 0.4 R_{200}$. Moreover, the ratio exhibits a constant increase with time, leading to a systematic underestimation of the predicted value of the RP with respect to the one measured at higher redshifts, $(z \gtrsim 1.5)$ for all the analysed halo mass ranges. These differences become more important for the innermost satellites $\left(r<0.5 R_{200}\right)$ of the most massive haloes, where the underestimation is present at any redshift, reaching even two decades at $z \sim 3$. This drawback of the T11 model is particularly troublesome if we consider that these satellites experience the highest values of RP during their evolution. Thus, this underestimation can have an important impact on the amount of gas stripped from the galaxies due to this environmental effect, changing the overall amount of gas available to form stars.

The resulting inaccurate behaviour can be explained by analysing the effect of the $P_{0}$ parameter on the prediction. This factor regulates the zero point of the RP profile inside the hosts, thus modulating the maximum value of $P_{\text {ram }}$ reachable towards their centres; a behaviour strongly dependent on the halo mass. Since the fit presented by T11 was constituted by several chi square minimizations, starting with the evolution in terms of the expansion factor of the Universe using linear models, the dependence with the mass was inadvertently softened creating an artificial bias. This bias can even be noticed in the upper panel of the Fig. 4 in T11, in which an example of the fit to the evolution of $P_{0}$ over the expansion factor is shown for a specific main host halo mass range. Even though the overlapped best linear fit follows the measured points and is always inside the estimated errors, this fit has a clear trend to underestimate the value of $P_{0}$ as redshift decreases, reaching differences with respect to the original value greater than one decade. It is clear that, even in that first T11 fitting process, the original trend of the parameter is lost and the prediction is softened due to the weight of the measurements in all the considered redshift range. Therefore, the biased behaviour of the T11 model was a result of the high number of
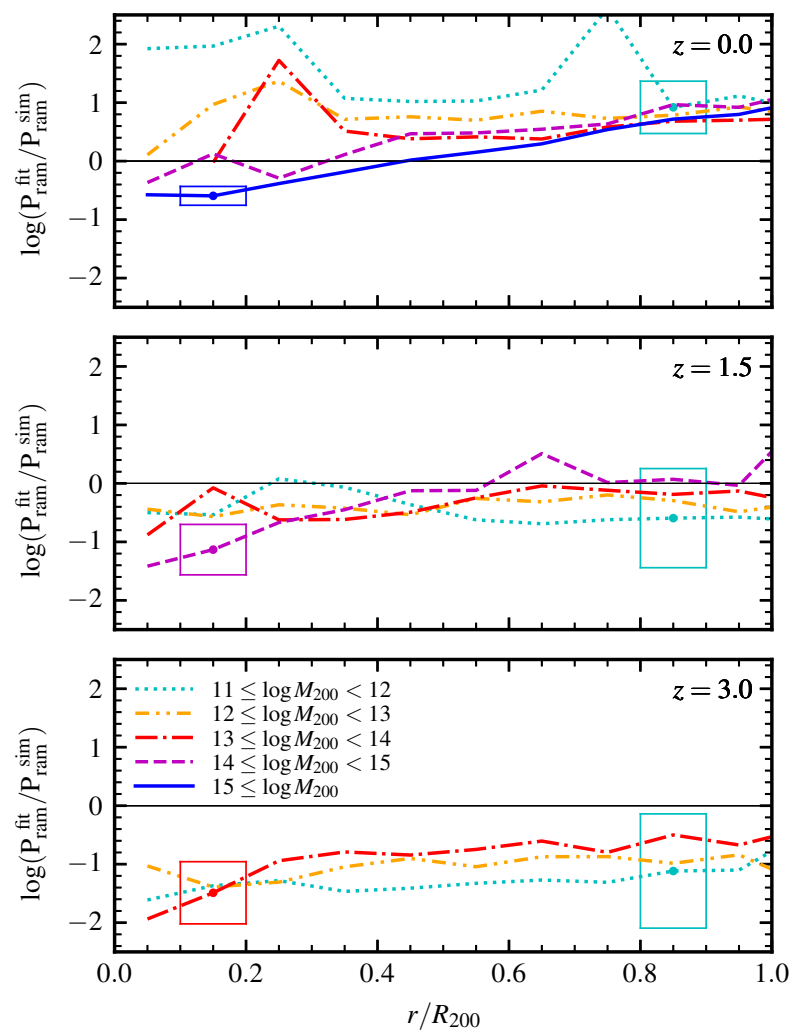

Figure 1. The mean ratio between the RP predicted by the T11 analytic model, $P_{\text {ram }}^{\text {fit }}$, and the RP measured from the gas particles of the simulations, $P_{\text {ram }}^{\operatorname{sim}}$, in logarithmic scale, for $z=0,1.5$ and 3.0 in the upper, middle and bottom panels, respectively. The measurements are shown with respect to the galactocentric distance normalised to $R_{200}$, grouped according to the instantaneous main host halo mass and shown with different line styles and colours, as detailed in the legend. Empty squares show the size of the chosen bin in the relative distance (horizontal) and one standard deviation around the mean (vertical), depicted at $r / R_{200}=0.15(0.85)$ for the most (least) massive halo sample, respectively.

free parameters used and the consecutive minimizations performed to complete the process. The possible degeneracies between the ten parameters and the high dispersion on the data preclude from finding a predictive model which can reproduce consistently the highest values of the RP inside massive main host haloes.

\section{NEW RAM PRESSURE PROFILE MODEL}

With the aim of having a more accurate model for the RP profile, we recreated the original $\mathrm{T} 11 \mathrm{fit}$, but also tried an alternative analytic version by inverting the order in which redshift and halo mass dependencies are modelled for the $P_{0}, r_{\mathrm{s}}$ and $\beta$ parameters. However, no improvements in the predictions were found with this exercise. Therefore, the profile as proposed by $\mathrm{T} 11$ does not constitute a model consistent for different epochs and halo masses.

In order to find a model with better agreement, a different approach must be taken. The described limitations forces us to provide an analytical description of the RP profiles capable of reproducing their behaviour for different halo mass ranges at a given redshift, using the minimum number of free parameters. Moreover, due to 

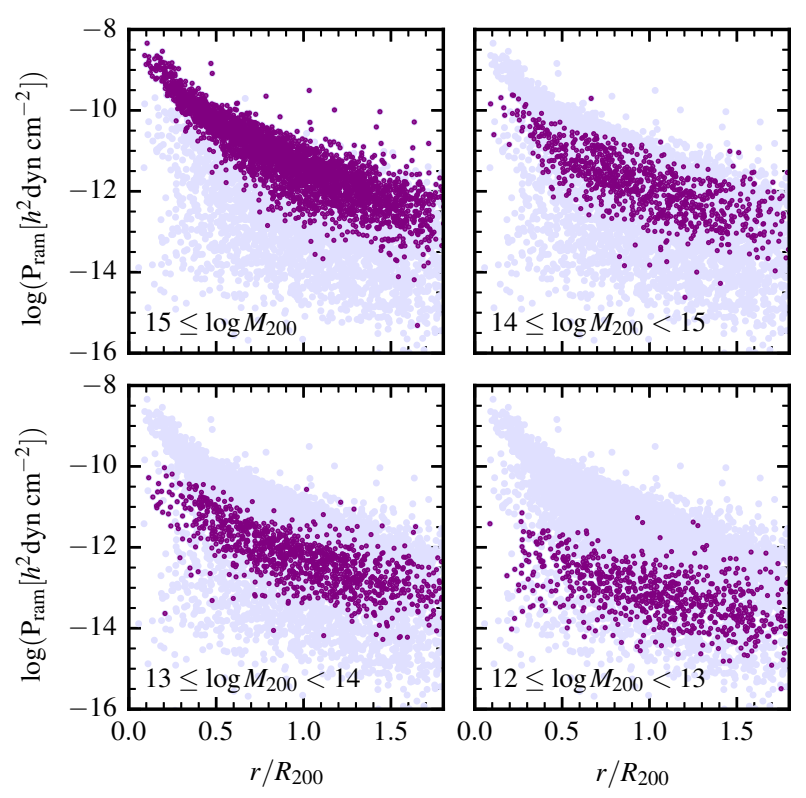

Figure 2. RP values obtained by applying the Tecce et al. (2010) technique on each satellite of the selected haloes from the resimulated regions at $z=0$, as a function of halocentric distance normalized with the virial radius of the halo, for different halo mass ranges. For reference, all panels depict the complete set of measurements with light purple dots. Each panel highlights, with dark purple dots, the measurements corresponding to the satellites from host haloes selected according to the mass range indicated in each legend.

the need of expressing the temporal evolution of those parameters, degeneracies between them must be avoided.

An example of the measured RP on the simulations, using the Tecce et al. (2010) technique, can be seen in Fig. 2. Here, the values of $P_{\text {ram }}$ for the complete sample of satellites found at $z=0$ are shown according to their relative distances to the centre of the haloes they reside in. In order to understand the main host halo mass dependence, all measurements are included in light purple dots, in all the four panels of the figure. Each panel highlights, with dark purple dots, the satellites that belong to a given mass range, as indicated on the key at the bottom.

According to this, the proposed expression should be able to follow the steep increment of the RP towards the centre of the massive haloes, which are the systems imprinting the RP in the stronger regime and have a higher impact in their star-forming satellites. The expression should also be able to reproduce simultaneously the flatter behaviour observed at different radial distances for the less massive haloes. This can be achieved with a profile defined by

$P_{\text {ram }}(M, z)=P_{0}(z)\left[\frac{1}{\xi(z)}\left(\frac{r}{R_{200}}\right)\right]^{-\frac{3}{2} \alpha\left(M_{200}, z\right)}$,

where $r / R_{200}$ is the relative distance of the satellite to the centre of the main host halo in terms of the virial radius, and $P_{0}(z), \xi(z)$, and $\alpha(M, z)$ are free parameters to define the shape of the profile. The $P_{0}(z)$ parameter, expressed in units of $h^{2}$ dyn $\mathrm{cm}^{-2}$, defines the normalization of the profile, whereas the dimensionless $\xi(z)$ determines the radial scaling, both dependent only on the redshift. The power $\alpha(M, z)$ encapsulates the dependence on the halo mass $M_{200}$ following a linear relation in logarithmic scale according to

$\alpha\left(M_{200}, z\right)=\alpha_{\mathrm{M}}(z) \log \left(M_{200} h^{-1}\left[\mathrm{M}_{\odot}\right]\right)+\alpha_{\mathrm{N}}$,

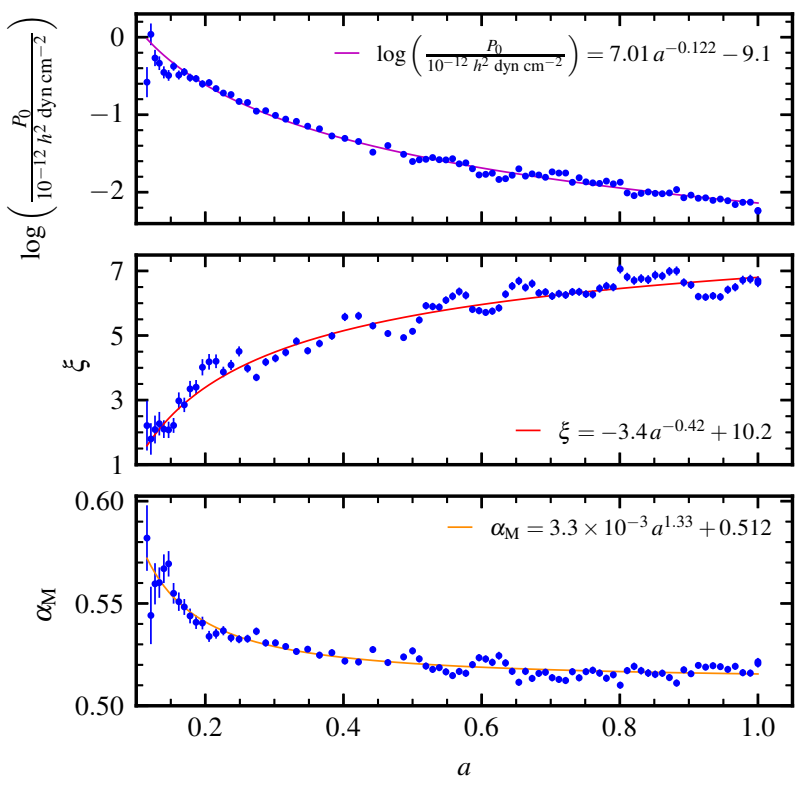

Figure 3. Temporal evolution, in terms of the scale factor $a$, of the $P_{0}(z)$ (upper), $\xi(z)$ (center), and $\alpha_{M}(z)$ (bottom) parameters of the introduced $\mathrm{RP}$ analytic profile. Blue dots show the numerical values found in the fitting process at each snapshot, with the error bars extracted from the diagonal elements of the covariant matrices. Solid coloured lines show the fitted model of each parameter. The corresponding analytic expression is indicated in each legend.

where $\alpha_{\mathrm{M}}(z)$ and $\alpha_{\mathrm{N}}$ are the free parameters to set the linear model of the power. To break the evident degeneracy between these last two parameters, a fixed value of $\alpha_{\mathrm{N}}=-5.5$ was chosen based on preliminary fits of this model to the data. By doing this, absolute minimums for the remaining three parameters is guaranteed in the minimisation processes.

Thereby, by using the equations (7) and (8) as a new model for the RP, we proceed to fit the three free parameters in each one of the resimulation snapshots through chi square minimizations. This is done simultaneously considering all the measurements of the RP at each redshift. The whole redshift range covered by the main host haloes of these simulations $(0 \leq z<7.5)$ is considered to perform the minimizations, instead of the restricted range $(0 \leq z \leq 3)$ adopted by T11. Furthermore, each minimization is performed including all the available range in halo mass covered by the complete set of resimulations. As described in the previous section, only the positions of the satellite haloes identified by the halo finder are used in this procedure to define the points to measure and fit the $\mathrm{RP}$ profile. It is worth noting that we do not apply any restriction in radial halocentric distance to the satellites of the systems considered in this fitting process. Approximately half of the satellites of each main host halo mass range shown in Figure 2 are lying in the external regions of their corresponding hosts, having distances larger than $R_{200}$. The measurements of the RP experienced by these more distant satellites allow tracing the shape of the profile in the outskirts of the haloes, where infalling systems can be affected by the medium. Note that our profile is defined only considering the halos that are assigned to the host according to the FOF results.

The resulting low degeneracy between the three fitted parameters can be seen in the smooth behaviour of the curves shown in Fig. 3. Here, the resulting numerical values obtained from all the 


\begin{tabular}{lllllll}
\hline Param. & $\lambda_{1}$ & $\delta \lambda_{1}$ & $\lambda_{2}$ & $\delta \lambda_{2}$ & $\lambda_{3}$ & $\delta \lambda_{3}$ \\
\hline $\log \left(P_{0}\right)$ & 7.01 & 3.0 & -0.122 & 0.047 & -9.1 & 3.02 \\
$\xi$ & -3.4 & 1.4 & -0.42 & 0.12 & 10.2 & 1.4 \\
$\alpha_{\mathrm{M}}$ & $3.3 \times 10^{-3}$ & $1.1 \times 10^{-3}$ & 1.33 & 0.18 & 0.512 & $1.6 \times 10^{-3}$ \\
\hline
\end{tabular}

Table 1. Resulting numerical values of the $\lambda$ coefficients which describe the temporal evolution of the $P_{0}, \xi$ y $\alpha_{\mathrm{M}}$ parameters defining the RP profile. The uncertainties were extracted directly from the obtained covariance matrices.

fits of the parameters that characterise the RP profile at each redshift are shown in blue dots, and each snapshot is shown in terms of the expansion factor of the Universe $a$. The reported errors, included as vertical lines around each dot, are extracted directly from the diagonal elements of the covariant matrices obtained from the fits, assuming no correlation between them. In the three cases, a very smooth temporal evolution of the numerical values is found. This is exactly the type of behaviour which allows to recover the desired predictability of the $P_{\text {ram }}$ model in the extreme cases of the massive haloes.

Finally, we model the temporal evolution of these parameters through simple power laws with respect to the expansion factor, including a shift in the vertical axis, according to

$\mathcal{P}=\lambda_{1} a^{\lambda_{2}}+\lambda_{3}$,

where $\mathcal{P}$ corresponds to any of the $\log \left(P_{0}\right), \xi$ and $\alpha_{\mathrm{M}}$ parameters, and the $\lambda_{i}$ with $i=1,2,3$ are the three free coefficients of the power law model associated to any one of them. The result of these three fits are also shown in Fig. 3 with solid coloured lines, and clearly smoothly follow the temporal evolution of the RP profile parameters (blue points). The final numerical values obtained for each one of the $\lambda_{i}$ coefficients are listed in Table 1 , together with their respective errors resulting from the chi square minimization processes.

According to the resulting values of the non diagonal elements of the covariance matrices obtained from the fits (not included in the table for simplicity), we find a degeneracy between the $\lambda_{1}$ and $\lambda_{3}$ coefficients. This is consistent with the reported errors associated to each coefficient, which are smaller than the observed amplitude of the little ripples exhibited by the curves. Nevertheless, since these values are explicitly included in the definition of the model, and they are not being fitted inside another relation, any degeneracy found at this level does not modify the resulting predictions for the RP model, thus becoming irrelevant.

As a proof of the better behaviour of this new model, we replicate the analysis described in the Section 2.3 but comparing this new fitted model with the measured RP profile from the resimulations. This comparison is shown in Fig. 4. As in Fig. 1, here we show the dependence of the ratio between the predicted values of RP given by the model and the measurements from the simulations, on halocentric distance normalized to $R_{200}$. It considers the location of satellites from different main host halo mass ranges at the same three redshifts previously analysed. According to the figure, the original reported bias was successfully avoided in this new model and its accuracy was substantially enhanced. Most of the artificial dependencies with the relative distance to the centre and halo mass featured by the T11 are not present in our new profile. A slight underestimation of the RP towards the centre of the most massive main host haloes at high redshifts, and a general overestimation towards the outskirts can be appreciated. However, these trends are statistically meaningless considering the natural spread of the measured data in the sample of haloes.

It is appropriate to take into account the dependence of our
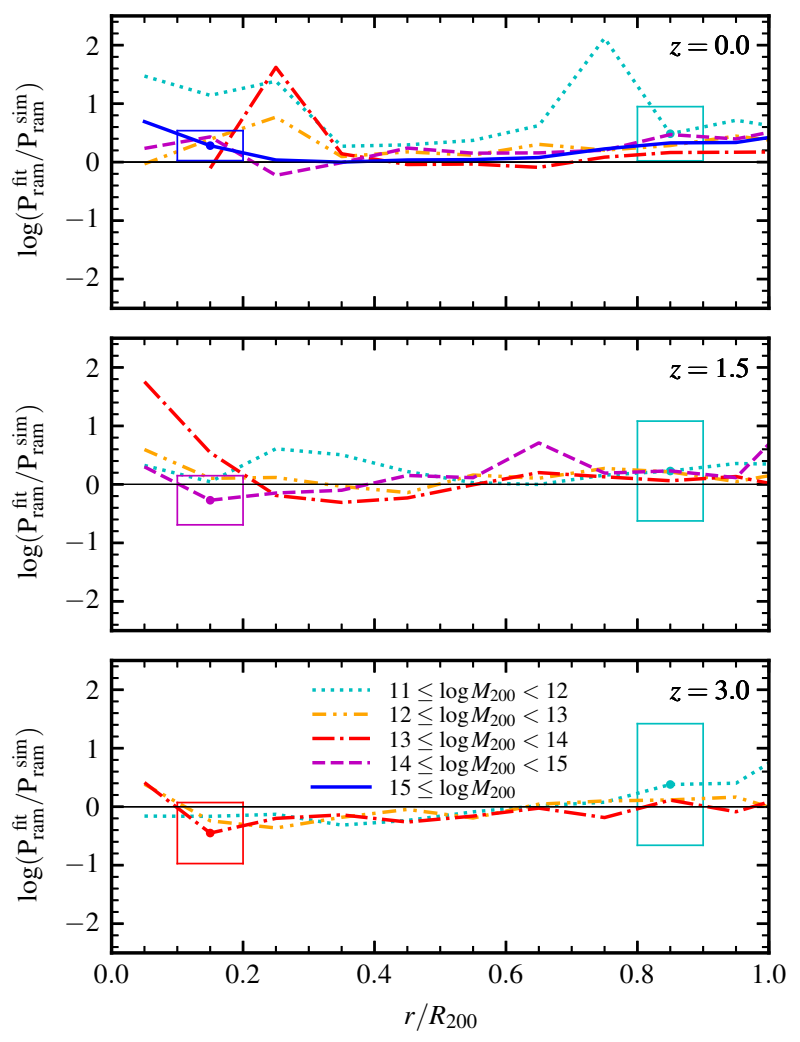

Figure 4. Comparison between the RP obtained with the new analytic model introduced in this work and the one measured from the gas particles of the simulations, for $z=0,1.5$ and 3.0 in the upper, middle and bottom panels, respectively. The symbols, binning and selection details are the same as in Fig. 1.

fitted profiles on the numerical method applied to model both the gas dynamics and the dark matter evolution in the cosmological simulations. Although it has been shown that the technique used to measure RP, when applied to this particular set of simulations, is able to recover ICM profiles that are consistent with X-ray observation of galaxy clusters (Tecce et al. 2010), it has also been reported that other techniques to model the hydrodynamics of the gas can yield differences in the bulk gas properties of clusters (Heß \& Springel 2012). Besides, different implementations for the modelling of the baryonic physics can affect the global properties of the gas content of simulated galaxies (e.g. Dolag et al. 2009; Davé et al. 2020), and also the dark matter halo properties (e.g. Beltz-Mohrmann \& Berlind 2021; Chua et al. 2021). On the other hand, comparisons between different techniques to simulate the hydrodynamics in combination with the relevant baryonic processes (like star formation and feedback) on galaxy clusters have shown that the influence of baryons on the total density profiles can be constrained within the innermost regions at $r / r_{500}<0.01-0.1$, depending on redshift (Mostoghiu et al. 2019), and they have also shown that the gas density at $r / r_{500}>0.2$ depends only weekly on baryon models ( $\mathrm{Li}$ et al. 2020). Based on these results, a revision of the fitted free parameters included in our proposed profile might be necessary to reproduce RP predictions from newer high-resolution full-physics simulations of galaxy formation, whose predictions of the hot gas density profiles or orbital evolution of satellites substantially differ, in particular towards the cluster centres. Accordingly, in a companion paper (Pallero et al. 2020), we evaluate our profile 
to model RP in galaxy clusters from the C-EAGLE simulations suit (Barnes et al. 2017), finding a general statistical agreement between the spherically averaged RP profiles measured from the particle distribution and our analytic model, without modifying its parameter values.

Accordingly, this new fit is predictive enough to model the RP at higher redshifts, in contrast to the capabilities of the T11 fit, and it can be used in different scopes to track the amount of gas being lost by satellite galaxies orbiting within their main host haloes. In the next section, we evaluate the impact of a consistent treatment of this environmental effect on satellite galaxies by comparing the results obtained from applying the new model of RP profile and the T11 fit.

\section{RAM PRESSURE IN GALAXY EVOLUTION}

In order to analyse the specific effect of RP on galaxy properties, a galaxy formation and evolution model must be applied in these simulations, including the stripping by RP.

\subsection{Semi-analytic model of galaxy evolution}

To create the galaxy populations from the simulations described in Sec. 2.1, we consider the updated version of the semi-analytic model of galaxy formation SAG (Cora 2006; Lagos et al. 2008; Tecce et al. 2010; Orsi et al. 2014; Muñoz Arancibia et al. 2015; Gargiulo et al. 2015; Cora et al. 2018). It uses the halo catalogues and merger trees to follow the evolution of the galaxy properties, assigning one galaxy to each detected subhalo and solving a set of analytic relations between the galaxy components across cosmic time.

Among the included physical processes, the model considers the radiative cooling of the hot halo gas, star formation (quiescent and in starbursts), and a detailed treatment of the chemical enrichment considering the contribution from stellar winds and different types of supernovae (Cora 2006). Thereby, feedback from these supernovae is also considered. It features an updated treatment of this feedback whose calculation includes an explicit dependence on redshift based on relations measured from full-physics hydrodynamical simulations, and it considers ejection of gas from the haloes to avoid excess of stellar mass at high redshifts (Cora et al. 2018). The model also follows the growth of massive black holes in the centre of galaxies, and their corresponding feedback which suppresses gas cooling (Lagos et al. 2008). Starbursts can be triggered by galaxy mergers and disc instabilities, contributing to the formation of galaxy bulges (Lagos et al. 2008; Muñoz Arancibia et al. 2015; Gargiulo et al. 2015). Environmental effects like tidal and RP stripping are also included in the model. It incorporates a detailed treatment for RPS considering the gas mass loss from both the discs and the hot gas halo, so that satellite galaxies are processed according to a gradual starvation scheme of their hot gas (Tecce et al. 2010; Cora et al. 2018). An additional model to analytically follow the orbital evolution of orphan satellite galaxies is also considered (Cora et al. 2018), as their positions within the main host haloes are relevant for a consistent calculation of environmental effects in those galaxies. Additionally, the free parameters included in the modelled relations are usually calibrated to a set of observed relations of galaxy properties, by using the Particle Swarm Optimisation technique (Ruiz et al. 2015).

The gradual starvation scheme to remove the hot gas of satellite galaxies after infall replaces the instantaneous removal usually applied in SAMs. This is a key ingredient to analyse the overall effect of RP acting on satellites residing in different environments. The gradual stripping of the hot gas is based on the Font et al. (2008) model, considering the estimations from McCarthy et al. (2008) and assuming a spherical distribution of the gas. At each timestep, the model calculates a satellite-centric radius $r_{\text {sat }}$ beyond which the gas is stripped using a dynamic time-scale (calculated as in Zentner et al. 2005), following the condition

$P_{\text {ram }}>\alpha_{\mathrm{RP}} \frac{G M_{\mathrm{sat}}\left(r_{\mathrm{sat}}\right) \rho_{\mathrm{hot}}\left(r_{\mathrm{sat}}\right)}{r_{\mathrm{sat}}}$,

where $\alpha_{\mathrm{RP}}$ is a geometrical constant, $M_{\mathrm{sat}}$ is the total satellite mass, $\rho_{\text {hot }}$ is the hot gas density of the satellite and $P_{\text {ram }}$ is the RP exerted over the satellite, measured directly using the gas particles of the the simulation (Tecce et al. 2010) or estimated from an analytic profile (Tecce et al. 2011). A general value of $\alpha_{\mathrm{RP}}=5$ is adopted in the model (Cora et al. 2018), chosen according to the analyses done by McCarthy et al. (2008). The calculation of $M_{\text {sat }}$ considers the contribution of the hot gas mass integrated until $r_{\mathrm{sat}}$, using a spherical isothermal density profile, $\rho_{\text {hot }}(r) \propto r^{-2}$.

When the ratio between the hot gas mass and the baryonic mass of a satellite galaxy decreases below 0.1 , RP can strip gas from the galaxy disc following the model of Tecce et al. (2010). The stripping radius is calculated by using the Gunn \& Gott (1972) condition

$P_{\text {ram }}>2 \pi G \Sigma_{\text {stars }}(r) \Sigma_{\text {cold }}(r)$,

where $\Sigma_{\text {stars }}$ and $\Sigma_{\text {cold }}$ are the surface densities of the stellar and gas components of the galaxy disc, respectively. Both are modelled with exponential profiles with the same scale-length, initially calculated from the spin and radius of the dark matter subhalo hosting the satellite galaxy.

In both cases of stripping (hot and cold gas), the removed mass and metals fractions are transferred to the hot gas of the galaxy identified as the central of the processed satellite (i.e. the intracluster/group medium), which is the central galaxy of the main host halo in most of the cases. Besides, it is worth noting that the RPS models included in SAG do not consider a treatment for the stripping of gas from the ejected reservoir resulting from supernovae feedback. We refer the reader to Cora et al. $(2018,2019)$; Collacchioni et al. (2018) (and references therein) for more detailed descriptions of all the physical processes implemented in SAG, including the environmental effects considered here.

\subsection{Modelling the galaxy populations}

We applied the galaxy formation model sAG to the complete set of resimulations of clusters of galaxies described in Sec. 2.1 to trace the evolution of the corresponding galaxy population. The processing of this particular suit of simulations with previous version of SAG is described in Cora et al. (2008), Tecce et al. (2010) and Tecce et al. (2011). The model considers the dark matter (sub)haloes and their corresponding merger trees as initial and boundary conditions to follow the evolution of galaxy properties. The calculation of the baryonic content of the subhaloes for tracing galaxy components must consider the fraction of mass contained in the gas particles. We recall that, in dark matter-only simulations, the total matter density of the Universe (i.e. dark matter and baryons) is represented by the particle distribution of the simulations. Hence, in our analysis when computing total masses of subhaloes, for being used by the SAM, their dark matter component is corrected by the corresponding cosmological baryonic mass fraction. Therefore, we only use the gas particle distribution to measure the local RP acting over the 
semi-analytic satellite galaxies associated to the corresponding dark matter haloes, by applying the Tecce et al. (2010) technique.

We create a fiducial galaxy population applying the full-physics SAG model described in Cora et al. (2018). It considers the values of the free parameters that were calibrated to a dark matter only simulation of $1 \mathrm{Gpc} / h$ of sidelength $\left(\mathrm{SAG}_{\beta} 1.3\right.$ variant detailed in Cora et al. 2018, 2019) instead of tuning a new set of parameter values for this new resolution limit and cosmology. The calculation of RPS considers the values of RP measured from the gas particle distribution of the simulation, $P_{\mathrm{ram}}^{\mathrm{sim}}$. Besides, to isolate the effect of RPS, we disable the mass loss of gas and stars due to tidal stripping processes. To validate this model variant, we inspect several galaxy properties in order to guarantee the consistency of the obtained galaxy population, including the total baryonic fraction of all the galaxies, the conditional stellar mass function and the conditional luminosity function (CLF) of satellites. The resulting CLFs feature a decreasing behaviour with an excess towards the fainter-end of the function, when using the Cora et al. (2018) parameter values in comparison with the results from Lan et al. (2016). Therefore, we increase the parameters related with the efficiencies of supernovae feedback to improve the CLFs shape. Although the break and slopes of the CLFs are recovered with this change, the simultaneous fitting of the normalization for all the main host mass ranges requires a complete exploration of the set of model parameters. Hence, as we aim to compare galaxies in specific main host mass ranges, we notice this caveat and proceed with a fair model-to-model comparison restricted to each main host mass range. Besides, a complete recalibration of the current model, specifically focused on this set of resimulations, constitutes a computationally demanding task that is outside the scope of this work.

Subsequently, we create two additional variants of the galaxy modelling by considering different analytic estimations of RP: the fit of the RP profiles introduced by Tecce et al. (2011); and the revisited fit presented in this work, described and analysed in the previous section.

As the three galaxy models are identical except for the RP calculation, the resulting number of satellite galaxies obtained for each run are equal due to the sAG processing strategy. The model applies a pre-processing to the halo merger trees in order to identify the merging haloes producing orphan galaxies, it calculates their orbits and defines their merging timescales. (for a more detailed description of this pre-processing, we refer the reader to Delfino et al. 2021). This pre-processing is completed before the properties of the stellar and gas content of the galaxies are calculated. Therefore, a direct comparison between the satellite populations of the same clusters can be done. Besides, it is worth noting that when comparing conditional luminosity and mass functions of satellite galaxies within main hosts in different mass ranges, the resulting number counts show no significant differences between the three model variants analysed throughout this section.

\subsection{Mass stripped by ram pressure}

To understand the general impact of the RP modelling, we create two samples of satellite galaxies according to the mass of the main host halo they belong to. A massive sample, containing satellites of simulated clusters with $\log \left(M_{200} h^{-1}\left[\mathrm{M}_{\odot}\right]\right)>15$. This sample contains the three main host haloes of the simulations g1, g8 and g51 (see Section 2.1). Satellites of this sample are expected to experience the strongest RP values, especially near their cluster centres. The second sample is composed of all the satellites that belong to main hosts haloes with $13 \leq \log \left(M_{200} h^{-1}\left[\mathrm{M}_{\odot}\right]\right)<14$, selected from

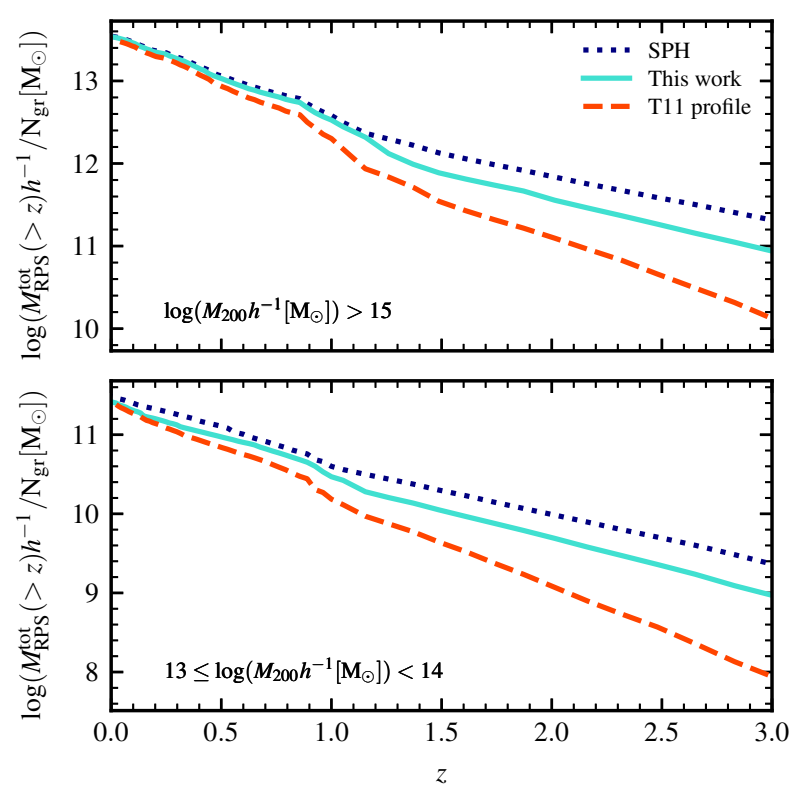

Figure 5. Mean cumulative function of stripped mass of the sample of satellite galaxies until a redshift $z$. The three model variants are depicted with different line styles and colours: the fiducial model in dotted dark blue lines, the model using the new RP profile in solid cyan lines, and the model using the T11 profile in dashed orange lines. Each function considers the complete population of satellites belonging to different ranges of main host halo: satellites from the 3 massive clusters (top panel) and from the selected 34 less massive main hosts with $13 \leq \log \left(M_{200} h^{-1}\left[\mathrm{M}_{\odot}\right]\right)<14$ (bottom panel).

the full set of resimulations. Considering only the non-contaminated haloes, this less massive sample includes the satellites belonging to 34 main host haloes.

We proceed to analyse these two samples in each of the three model variants. For an overall measuring of the RP effect, we take into account all the progenitors of the galaxies belonging to each $z=0$ selected halo (i.e. the complete set of members of their merger trees) and measure the total amount of gas mass stripped by $\mathrm{RP}$ at each simulation timestep. This allows to define the cumulative function of stripped mass, $M_{\mathrm{RPS}}(>z)$, as the sum of all the stripping measurements from the progenitors of a selected galaxy until a given redshift, $z$. It includes the gas stripped from both the gas disc and hot gas halo components to quantify the complete effect of this environmental process. As a result, $M_{\mathrm{RPS}}(>z=0)$ corresponds to the total mass that a galaxy has lost by RPS until $z=0$, accounting all its progenitors. Combining the results obtained from all the selected galaxies, we calculate the cumulative function of stripped mass of the complete sample, at any redshift, as the sum

$M_{\mathrm{RPS}}^{\mathrm{tot}}(>z)=\Sigma_{i} M_{\mathrm{RPS}}^{i}(>z)$,

where $i$ refers to the selected galaxies of the sample. Finally, we obtain the mean by dividing by the number of main host haloes selected in each sample, $\mathrm{N}_{\mathrm{gr}}$. The resulting function is shown in Fig. 5. The top panel shows the results of the satellite sample from the higher main host mass, whereas the bottom panel shows the results for the lower mass sample. Each panel shows the three model variants: the dotted dark blue lines show our fiducial model considering the RP as measured from the SPH simulation, the solid cyan lines show the model using our new analytic RP profile, and the dashed orange lines show the model using the T11 fit. 
For both mass ranges, the total amount of gas mass stripped tends to reach the same value at $z=0$, independently of the RP model. The values are approximately equivalent to the 2.3 and 0.8 per cent of the mean main host total mass for the high and low mass samples, respectively. Therefore, this global stripping process seems sightly more significant towards higher cluster masses; an expected behaviour as the RP acting on satellites near the centre of their hosts is able to reach larger values when the mass of the main host is high (e.g. Wetzel et al. 2012; Haines et al. 2015). Nonetheless, it should be warned that the effective final RP stripped mass amount has a direct dependence on the general modelling of galaxy evolution, i.e. the physical processes considered in the semi-analytic model and the corresponding calibration of its free parameters. Besides, as the number of satellites and total mass of each system increases over cosmic time, the amount of mass involved in newer stripping events becomes more significant than the older ones in this figure. This also contributes to the similar behaviour of the accumulated RP stripped mass exhibited by the three model variants. On the other hand, the small knee near $z \sim 1$ featured by all models only appears when using the larger parameters related with the supernovae feedback process in the SAM, but is not present with the original calibrated parameter set. Thus, this break is a direct consequence of the physical relation introduced to model the supernovae feedback, as its efficiency has a strong dependence on redshift and changes its dependence on the halo virial velocity when this property reach $60 \mathrm{~km} / \mathrm{s}$ (see Cora et al. 2018, equations (10) and (12)).

The general trends of the two mass bins are analogue. The model which applies the RP profile described in this work is more effective in stripping mass than the one described by T11, particularly at high redshift. We note that, although our proposed RP profile represent a significant improvement with respect to $\mathrm{T} 11$, there are still some systematic differences with respect to the fiducial model. The diversity in the dynamics of the infalling satellites plays a role here. Although our analytic model is able to reproduce the increasing RP values towards the centre of the haloes (see Fig. 4), a small fraction of satellites with larger galactocentric distances can also exhibit comparable RP values than those near the centre (as it can be observed from the vertical spread of the measurements shown in Fig. 2) due to their high velocities relative to the host (e.g. see Oman et al. 2013). Consequently, the RP felt by these objects can not be reproduced by the analytic models, as these satellites are outliers of the mean trends. Besides, our fitting profile assumes a spherically symmetric distribution of the gas within main host haloes, so that large values of RP resulting from inhomogeneities are not considered. According to this, the analytic fit constitutes a good approximation to the median values of RP, but it must be noted that a fraction of the extreme cases of stripped galaxies can not be fully recovered with this method. This is in line with some of the expected limitations associated with semi-analytic modelling of galaxy evolution.

Besides these extreme cases, the new modelling of RP profiles can have a non-negligible impact on the properties of the global population of satellites, in particular on satellites residing in high density environments where the median RP is higher. Therefore, the following analysis will be focused in the properties of this galaxy population.

\subsection{Ram pressure on cluster galaxies}

To quantify the effective impact of the RP on individual galaxies, we define the instantaneous fraction of the total stripped mass, $f_{\text {RPS }}$, as the ratio between the total stripped mass of a satellite and its stellar mass, i.e.

$f_{\mathrm{RPS}} \equiv \frac{M_{\mathrm{RPS}}\left(>z=z_{i}\right)}{M_{\star}\left(z_{i}\right)}$,

where $M_{\mathrm{RPS}}\left(>z=z_{i}\right)$ is the total stripped mass of a satellite until a redshift $z_{i}$, as defined in the previous section, and $M_{\star}\left(z_{i}\right)$ is its instantaneous stellar mass at $z_{i}$. The $f_{\mathrm{RPS}}$ distributions exhibited by the complete sample of satellites belonging to the three most massive clusters are analysed by counting the number of galaxies having different values of this quantity per cluster unit. To analyse the distributions at different redshifts, we consider the progenitors of the selected $z=0$ main host haloes, and calculate $M_{\mathrm{RPS}}\left(>z=z_{i}\right)$ for all their satellites using their respective merger trees, accordingly. Hence, $\mathrm{N}_{\mathrm{gr}}=3$ in this analysis. At each redshift in the fiducial model, we select all satellites having $\log \left(M_{\star}\left[\mathrm{M}_{\odot}\right]\right) \geq 8$, being this limit chosen according to the behaviour of the conditional stellar mass functions of satellites at different redshifts which start to misbehave for stellar masses below $\sim 10^{7} \mathrm{M}_{\odot}$. We can confidently consider this limit due to we are analysing satellite galaxies from a model that includes a consistent treatment for the orphan galaxies, so we can compare low mass satellites processed by the environmental effects. We then identify the haloes of this selected galaxies and use them to identify the corresponding galaxies in the remaining two models for a direct comparison. The resulting function is shown in the Fig. 6. We consider three distinct epochs, $z=0.0,1.5$ and 3.0 shown in the top, middle and bottom panels, respectively. The selection of redshifts considers the same values and time range as the analysis done in the previous sections. The distribution of number counts resulting from the fiducial model using the values of $\mathrm{RP}$ measured from the SPH simulation is shown with the filled area and the dotted dark blue line, whereas the solid cyan line and the dashed orange line depict the number counts of the model applying our new profile and the model using the T11 fit, respectively (as in Fig. 5). In addition, the small vertical lines located in the upper axis of each panel indicates the median values of each distribution. These medians clearly follow the same trend as the cumulative functions of stripped mass shown in Fig. 5, where lower fractions at any epoch result when analytic RP profiles are applied. In particular, the T11 fit produces noticeably lower median fractions as redshift increases, reaching differences of almost 1.5 decades at $z=3.0$ with respect to the fiducial model results. Moreover, the global shape of the distributions of the model applying the T11 profile are skewed to lower values of $f_{\text {RPS }}$ as redshift increases, indicating a persistent smaller fraction of stripped mass in the complete satellite galaxy population. The analytic fit presented in this work features a noticeable improvement in the modelling of the number of galaxies with large fractions of stripped mass although, as previously discussed, is unable to fully recover the total number of galaxies with larger fractions.

An interesting feature of the distributions shown in Fig. 6 is the global increasing mean values of $f_{\mathrm{RPS}}$ over time, exhibited by the three variants of the galaxy formation model. As redshift decreases, there is an increasing number of satellite galaxies whose accumulated total mass lost by RPS is larger than their instantaneous stellar mass (i.e. $\log \left(f_{\text {RPS }}\right)>0$ ). This is interesting considering that the stellar mass of these galaxies is not being reduced by external processes (like tidal stripping) besides stellar evolution. To further analyse the behaviour of $f_{\text {RPS }}$, in Fig. 7 we show the median values obtained from the $z=0$ satellite sample, considering the three model variants. Satellites are binned according to their stellar masses. The same colour coding as the previous figure is used, but here the shaded area and the thin lines depict the percentiles 10 and 90 of 


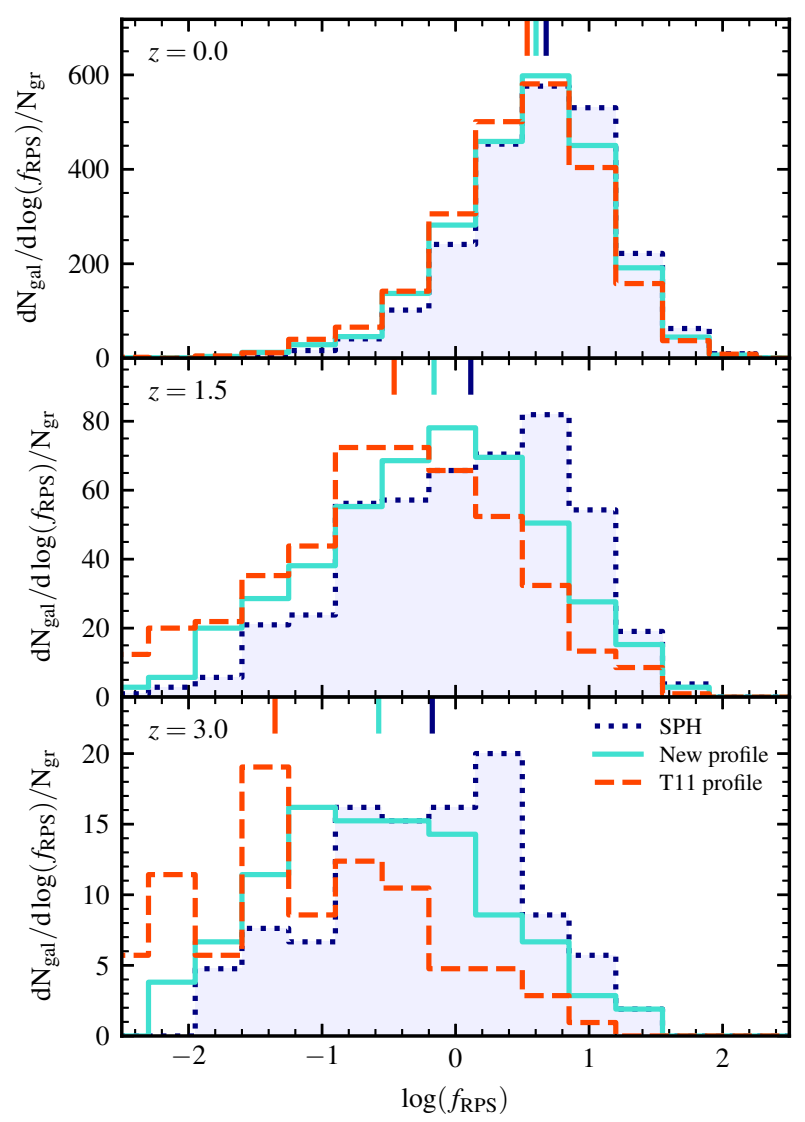

Figure 6. Number counts of satellite galaxies having different values of their instantaneous fraction of total stripped mass, defined by equation (13). Each panel shows a different redshift: $z=0,1.5$ and 2 in the top, centre and bottom panels, respectively. Fiducial model resulting distributions are depicted with dotted dark blue lines and shaded areas, whereas the new and T11 profiles are depicted with solid cyan and dashed orange lines, respectively. Median values are shown with small vertical lines at the top of each panel using the same colour coding.

the measurements in each stellar mass bin. In general, the median $\log \left(f_{\mathrm{RPS}}\right)$ shows a constant increase with decreasing stellar masses for galaxies having $\log \left(M_{\star}\left[\mathrm{M}_{\odot}\right]\right) \lesssim 10.4$, mass above which the trends feature a turn-up and start to increase towards higher stellar masses. It is interesting to note that the change in the trends is parallel in the three model variants. However, we can not draw robust conclusions from this higher mass range as the number of satellites having $\log \left(M_{\star}\left[\mathrm{M}_{\odot}\right]\right)>10.4$ constitute less than a 0.09 fraction of the selected sample in each model variants. Furthermore, their measured $f_{\text {RPS }}$ show a significant dispersion. In general, we find that galaxies featuring the largest values of $\log \left(f_{\text {RPS }}\right)$ have low stellar masses, in the mass regime corresponding to dwarf galaxies. Therefore, these galaxies are more susceptible to be affected by $\mathrm{RP}$, in agreement with previous results obtained with the model (Cora et al. 2018, 2019), and other studies (e.g. see Roberts et al. 2019).

To go further in the analysis, now the main properties of the population of satellites with the largest fractions of stripped mass are compared. At each redshift, a cut in $f_{\mathrm{RPS}}$ following the median value obtained from the fiducial model is chosen to select the galaxies. The same cut applied to the three model variants allows us to compare galaxies that are being affected with analogue stripping

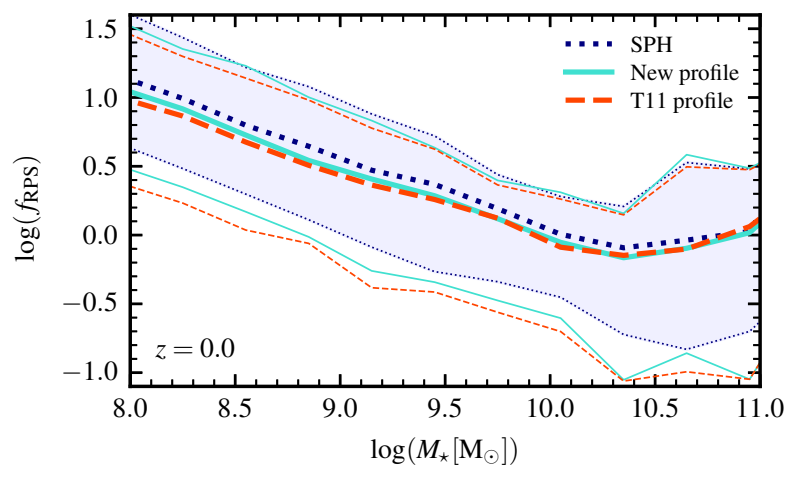

Figure 7. Median values of the instantaneous fraction of total stripped mass of the satellite galaxies of the massive clusters at $z=0$, as a function of their stellar mass. Fiducial model results are depicted with dotted dark blue lines and shaded area, whereas the new and T11 profiles are represented by solid cyan and dashed orange lines, respectively. Median values are shown with thick lines, whereas the shaded area and thin lines depict the percentiles 10 and 90 of the measurements in each stellar mass bin.

processes. Thereby, differences in the number counts of galaxies allow measuring the overall effect of using the analytic models to estimate RP. Median values, indicating the half of the distributions, are preferred instead of a larger fraction only for visualisation purposes. This guarantees a number of galaxies large enough to be compared in all considered redshifts. It is worth noting that trends and general conclusions reported in this section are not affected when the cut applied in the fraction is increased (e.g. using the value defining the highest quartile of the distribution instead of the median). Therefore, the chosen limits in $f_{\text {RPS }}$ to select the galaxy samples are 4.78, 1.30 and 0.67 for redshifts $0.0,1.5$ and 3.0, respectively.

The comparison between the selected galaxies properties from the three model variants is shown in Fig. 8. The differential galaxy number counts according to their stellar mass, specific star formation rate and $(g-r)$ rest-frame colour are shown in the left, middle and right columns, respectively. The comparison includes the three considered redshifts in different rows, as indicated on each panel. The colour coding of the models is the same as in Fig. 6 . The general trend already spotted before remains in all the cases presented here. This is, the number of galaxies having the largest amounts of stripped mass are lower in the models using the analytic profiles of RP than in the fiducial one, being the T11 profile the one featuring the lowest amount of galaxies with large stripped mass fractions. The distributions of number counts of galaxies with different stellar masses show that most of the satellite population experiencing the larger fractions of total stripped mass, and not recovered when the analytic fits are applied, tend to have low stellar mass $\left(M_{\star} \leq 10^{9.5} \mathrm{M}_{\odot}\right)$, in the regime of dwarf satellite galaxies. This trend is noticeably larger at high redshifts for the T11 model, in agreement with the reported systematic strong subestimation of the general $P_{\text {ram }}$ modelling exhibited by that profile. As redshift decreases, the relative impact of the RPS process into the final stellar mass distribution seems to become milder as the satellite mass distributions progressively become more similar for the different models. Besides, the total number of cluster satellites at $z=0$ is considerably larger than at high redshifts, and a significant fraction of these satellites is expected to be accreted as part of less massive groups (McGee et al. 2009; Pallero et al. 2019, 2020). Hence, the stripping of gas experienced by these galaxies has a non negligible contri- 

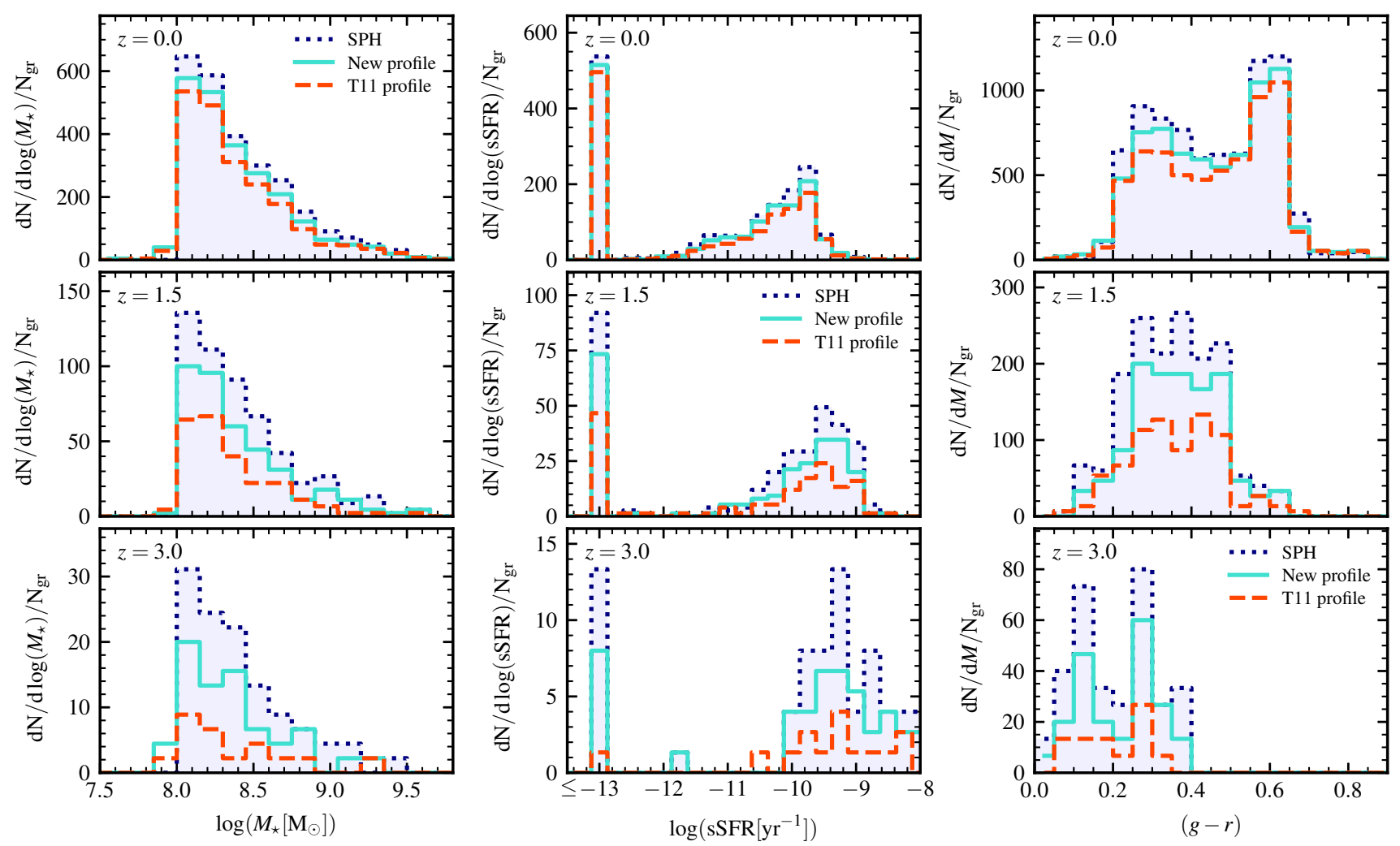

Figure 8. Differential number counts of galaxies binned by: stellar mass (left panels), specific star formation rate (centre panels), and ( $g$ - $r$ ) colour (right panels). Three different epochs are considered: $z=0$ (top panels), 1.5 (middle panels), and 3.0 (bottom panels). At each redshift, the sample of galaxies is selected by applying a cut in their instantaneous fraction of total stripped mass, $f_{\text {RPS }}$. The selected value for this cut is chosen according to the median in the distribution of $f_{\mathrm{RPS}}$ as found in the model of RPS measured from the SPH simulation: $4.78,1.30$ and 0.67 for redshifts $0.0,1.5$ and 3.0 , respectively.

bution from the environment they inhabited before being accreted by the cluster. On top of that, the small tail in the low mass limit, featured by the models using the analytic RP profiles, points towards a general decrease of the resulting galaxy masses, in comparison with the fiducial model results. Moreover, as we are comparing the galaxies with the largest amounts of stripped mass obtained with each model variant, the trends exhibited at each redshift suggest that the general effect of the stripping process has a stronger impact on low-mass galaxies, in agreement with results derived from observed galaxy properties (Haines et al. 2006; Bamford et al. 2009; Peng et al. 2010; Roberts et al. 2019).

As the stripping of gas from discs by RP is characterised by short time-scales (e.g. Foltz et al. 2018), in Fig. 8 we also compare the galaxies sSFR and $(g-r)$ colour number count distributions obtained from the three model variants, to analyse the more instantaneous impact of this environmental effect on their properties. The sAG model does not consider star formation triggered by gas compression occurring during stripping events, so galaxies with high SFR in the sample have their star formation strictly driven by internal processes or galaxy mergers. The sSFR of each galaxy is calculated considering all newly formed stars in the time elapsed between two consecutive snapshots of the simulation, approximately 200 Myrs, divided by their stellar mass. All the quenched satellites having values of sSFR lower than $10^{-13} \mathrm{yr}^{-1}$ are manually included in the lowest bin of the distributions as indicated in the corresponding axis label of the figure for visualisation purposes. The selected sample shows distributions that continuously tend to have lower star formation as redshift decreases. Thereby, using the commonly applied $z=0$ threshold, sSFR $=10^{-11} \mathrm{yr}^{-1}$ (e.g. Wetzel et al. 2012), as a simplistic approach to separate star-forming from quiescent galaxy populations on the three analysed redshifts (as in Fontanot et al. 2009), the resulting number of quenched galaxies experiencing the largest gas stripping increases over time. It is however important to note that this threshold does not take into account the evolution of the sSFR distribution over redshift, being unable to properly separate the quenched population at $z>0$ (e.g. Fang et al. 2018; Donnari et al. 2019). As a result, our chosen threshold only serves as a reference value for comparing number counts of galaxies in different sSFR regimes. According to this, the resulting number of quiescent galaxies exhibits the most important differences among the model variants, being the model using the T11 profile the one that features the largest lack of quenched galaxies at higher redshifts in comparison with the fiducial galaxy sample. Nonetheless, like the distributions of stellar mass, the resulting distributions of $\mathrm{sSFR}$ at $z=0$ of the three model variants are similar. The observed differences at high redshifts, resulting from the RP modelling, are masked at $z=0$ because of the increased number of satellites. Besides, as a fraction of these satellites are accreted within less massive groups, their star formation histories may have been preprocessed in different environments outside the virial radius of the cluster (Pallero et al. 2019), leading to different stripping scenarios during their evolution. On the other hand, as redshift increases, the difference in the number of both quenched and star-forming galaxies becomes more important between the model variants. According to 
the results from the selected sample of galaxies, the star formation rate of satellites that have experienced large amounts of RPS is highly affected by this environmental effect at higher redshifts, even until $z \sim 3$, as the number of quiescent satellites substantially differs between the different approaches of RP modelling. It is worth noting that when the sSFR comparison at $z=3.0$ is shown instead using normalised functions, the resulting distributions feature the same trend spotted here, that is the fraction of galaxies having sSFR $\leq 10^{-13} \mathrm{yr}^{-1}$ is significantly lower when the T11 RP profile is applied in the calculation of the stripping mass. It has been previously stated that the role of environment on galaxy quenching in high density regions becomes important only after $z \sim 1.6$ (Nantais et al. 2016, 2017). This, however, does not disagree with the evolution of the sSFR exhibited by our sample as these galaxies are chosen according to their ratio of stripped mass, which determines a biased selection. Besides, as the sample is not compared with analogue field galaxies, the environmental quenching efficiency (Peng et al. 2010, 2012), usually estimated to evaluate the role of the environment on galaxy quenching, can not be calculated using this suit of resimulations.

The distributions of galaxy number counts of rest-frame $(g-r)$ colours of the selected galaxies are shown in the right panel of Fig 8. As in the case of the sSFR, differences in galaxy colours allow to evaluate the more instantaneous effect of RP in galaxies. The $z=0$ colours of the sample exhibit a clear bimodal distribution, with a redder peak slightly shifted $\sim 0.15$ mag in comparison with the usually observed red population of galaxies (e.g Bell et al. 2003; Taylor et al. 2015). However, as we are using a shallow calibration of the parameters of the SAG model, the global reported colours of the galaxies could be affected by a systematic shift. The distributions nonetheless show a clear evolution across redshift, being the galaxies affected by high RP more star-forming and, consequently, bluer with increasing redshift. Moreover, no difference indicating preferred colours are found among the different models. The lack in the number of galaxies resulting from the analytic models of $\mathrm{RP}$ is observed in the complete range of colours, being this galaxy property insensitive to the RP modelling.

According to these results, our new profile for modelling the $\mathrm{RP}$ exerted by galaxy groups and clusters exhibits a significant improvement in the general RP calculation in galaxy formation models, specifically for high redshift galaxies. The number of galaxies experiencing large amounts of RPS depends strongly on the RP modelling, and a smaller number of these galaxies are obtained with the T11 profile. Major differences are also found in galaxy properties, particularly in their stellar mass and star formation rate. We remark that these differences must be taken as a lower limit as the SAG model does not consider star formation induced in the stripped gas by RP, therefore such new populations of stars formed in these galaxies are missing in our analysis, a feature commonly observed in extremely stripped galaxies (e.g. Poggianti et al. 2016). Further analysis focused in this specific set of galaxies using improved simulations of galaxy clusters will allow to study more precisely the effect of the stripping events on global galaxy properties.

\section{SUMMARY AND CONCLUSIONS}

Using a set of SPH resimulations of galaxy clusters, we have analysed different approaches to model analytically the RP experienced by satellite galaxies inhabiting main host haloes whose masses span from less massive groups $\left(M_{200} \sim 10^{13} \mathrm{M}_{\odot}\right)$ to galaxy clusters $\left(M_{200} \sim\right.$ few $\left.10^{15} \mathrm{M}_{\odot}\right)$. We have also considered a large redshift range, from $7.5>z \geq 0.0$. The T11 RP profile (Tecce et al. 2011) was revisited in detail, showing misleading predictions at different epochs in comparison with the effective RP measured from the simulations using the Tecce et al. (2010) method. In addition, a new universal analytic model for the RP was introduced, and the impact of the application of this type of treatment in galaxy formation models was evaluated, focusing specifically on satellites residing in high density environments. The main results of these analysis can be summarised as follows:

- The T11 analytic RP profile features a systematic underestimation of the RP measured in the simulations where the profile was defined, reaching even 2 decades at $z=3$ in all the analysed main host masses. This underestimation persists towards the centres of massive galaxy clusters at $z=0$, however it reverses with increasing relative radial distance, reaching 1 decade of overestimation near $R_{200}$. The same trend but milder is present in the less massive haloes.

- Although T11 model is able to fit individual profiles of RP in haloes with different masses and from different epochs, a temporal or mass dependence of its free parameters can not be defined in order to set an universal analytic model of RP. Moreover, by doing so, as in Tecce et al. (2011), the analytic profile obtained from the fitting processes tends to flatten the predicted RP in radial distance, missing the largest and homogeneous values of RP experienced by the satellites close to the centres of massive hosts.

- We introduced a new analytic model for the RP profile which recovers the expected values measured from the simulation for a large range of main host halo masses, epochs and radial distances. The profile, defined by the equation (7), is characterised by a power which is a function of the main host halo mass through (8), i.e.

$P_{\text {ram }}=P_{0}(z)\left(\frac{r}{\xi(z) R_{200}}\right)^{-\frac{3}{2}\left[\alpha_{\mathrm{M}}(z) \log \left(M_{200} h^{-1}\left[\mathrm{M}_{\odot}\right]\right)-5.5\right]}$,

and the temporal evolution of the three free parameters were fit to the simulation RP measurements, giving

$$
\begin{aligned}
\log \left(P_{0} /\left(10^{-12} h^{2} \text { dyn }^{-2}\right)\right) & =7.01 a^{-0.122}-9.1, \\
\xi & =-3.4 a^{-0.42}+10.2, \\
\alpha_{\mathrm{M}}=3.3 & \times 10^{-3} a^{1.33}+0.512,
\end{aligned}
$$

expressed in terms of the scale factor $a$.

- By using a semi-analytic model of galaxy formation, we showed that the analytic fit proposed in this work to model the RP experienced by satellite galaxies represent a significant improvement with respect to the T11 model, specially at high redshifts $(z \gtrsim 1)$. We note, however, that some systematic differences still persist when comparing with the model using RP values measured from the underlying simulation. These differences are an expected limitation of the RP modelling when using analytic profiles.

- In high-density environments like galaxy clusters, where extreme high values of RP can be reached, differences among the models of RP are larger with increasing redshift. The comparison of the distribution of ratios between the accumulated stripped mass and the stellar mass of the satellites shows an increasing reduction with increasing redshift of the number of galaxies experiencing larger gas stripping when the T11 model is applied. Moreover, satellites experiencing the largest amounts of total stripped mass tend to have low stellar mass $\left(M_{\star} \leq 10^{9.5} \mathrm{M}_{\odot}\right)$ and their sSFR is dependent on the RP modelling applied, particularly at high redshifts.

In summary, according to the analysis of galaxy properties of satellites being evolved using three variants to model RP affecting 
them, our new profile exhibits a large improvement in the overall treatment of this environmental process, specially at high redshifts $(z>1)$.

\section{ACKNOWLEDGEMENTS}

The authors acknowledge the anonymous reviewer for the valuable comments and suggestions that contributed to improve the presentation of this research. CVM acknowledges support from ANID/FONDECYT through grant 3200918. CVM and FAG acknowledges financial support from the Max Planck Society through a Partner Group grant. FAG acknowledges support from ANID FONDECYT Regular 1181264. SAC acknowledges funding from Consejo Nacional de Investigaciones Científicas y Técnicas (CONICET, PIP-0387), Agencia Nacional de Promoción de la Investigación, el Desarrollo Tecnológico y la Innovación (Agencia I+D+i, PICT-2013-0317, PICT-2018-03743), and Universidad Nacional de La Plata (G11-150), Argentina. TH acknowledge CONICET, Argentina, for their supporting fellowships.

\section{DATA AVAILABILITY}

Most of the data referred through this article are publicly available in a dedicated repository in Githus at https://github.com/cnvega/PramFits. It includes the codes and scripts developed for analysis and figures, additional figures comparing both RP profiles with the measurements in each simulation snapshot, the resulting parameter values and covariance matrices of the RP profile fitting process, and the analysed galaxy catalogues of the three model variants. The raw data of the resimulations and the semi-analytic model of galaxy formation will be shared on reasonable request to the corresponding author.

\section{REFERENCES}

Abadi M. G., Moore B., Bower R. G., 1999, MNRAS, 308, 947

Ayromlou M., Nelson D., Yates R. M., Kauffmann G., White S. D. M., 2019, MNRAS, 487, 4313

Ayromlou M., Kauffmann G., Yates R. M., Nelson D., White S. D. M., 2021, MNRAS, 505, 492

Bahé Y. M., McCarthy I. G., 2015, MNRAS, 447, 969

Bahé Y. M., McCarthy I. G., Crain R. A., Theuns T., 2012, MNRAS, 424, 1179

Baldry I. K., Glazebrook K., Brinkmann J., Ivezić Ž., Lupton R. H., Nichol R. C., Szalay A. S., 2004, ApJ, 600, 681

Baldry I. K., Balogh M. L., Bower R. G., Glazebrook K., Nichol R. C., Bamford S. P., Budavari T., 2006, MNRAS, 373, 469

Bamford S. P., et al., 2009, MNRAS, 393, 1324

Barnes D. J., et al., 2017, MNRAS, 471, 1088

Bekki K., 2009, MNRAS, 399, 2221

Bekki K., 2014, MNRAS, 438, 444

Bekki K., Couch W. J., Shioya Y., 2002, ApJ, 577, 651

Bell E. F., McIntosh D. H., Katz N., Weinberg M. D., 2003, ApJS, 149, 289

Beltz-Mohrmann G. D., Berlind A. A., 2021, arXiv e-prints, p. arXiv:2103.05076

Bond J. R., Cole S., Efstathiou G., Kaiser N., 1991, ApJ, 379, 440

Boselli A., Gavazzi G., 2006, PASP, 118, 517

Boselli A., et al., 2016, A\&A, 596, A11

Cassata P., et al., 2008, A\&A, 483, L39

Chua K. T. E., Vogelsberger M., Pillepich A., Hernquist L., 2021, arXiv e-prints, p. arXiv:2109.00012
Churazov E., Forman W., Vikhlinin A., Tremaine S., Gerhard O., Jones C., 2008, MNRAS, 388, 1062

Collacchioni F., Cora S. A., Lagos C. D. P., Vega-Martínez C. A., 2018, MNRAS, 481, 954

Cora S. A., 2006, MNRAS, 368, 1540

Cora S. A., Tornatore L., Tozzi P., Dolag K., 2008, MNRAS, 386, 96

Cora S. A., et al., 2018, MNRAS, p. 1074

Cora S. A., Hough T., Vega-Martínez C. A., Orsi Á. A., 2019, MNRAS, 483, 1686

Darvish B., Mobasher B., Sobral D., Rettura A., Scoville N., Faisst A., Capak P., 2016, ApJ, 825, 113

Davé R., Crain R. A., Stevens A. R. H., Narayanan D., Saintonge A., Catinella B., Cortese L., 2020, MNRAS, 497, 146

Davis M., Efstathiou G., Frenk C. S., White S. D. M., 1985, ApJ, 292, 371

De Lucia G., Weinmann S., Poggianti B. M., Aragón-Salamanca A., Zaritsky D., 2012, MNRAS, 423, 1277

Delfino F. M., Scoccola C. G., Cora S. A., Vega-Martinez C. A., Gargiulo I. D., 2021, arXiv e-prints, p. arXiv:2102.01837

Dolag K., Vazza F., Brunetti G., Tormen G., 2005, MNRAS, 364, 753

Dolag K., Borgani S., Murante G., Springel V., 2009, MNRAS, 399, 497

Donnari M., et al., 2019, MNRAS, 485, 4817

Fang J. J., et al., 2018, ApJ, 858, 100

Fillingham S. P., Cooper M. C., Wheeler C., Garrison-Kimmel S., BoylanKolchin M., Bullock J. S., 2015, MNRAS, 454, 2039

Foltz R., et al., 2018, ApJ, 866, 136

Font A. S., et al., 2008, MNRAS, 389, 1619

Fontanot F., De Lucia G., Monaco P., Somerville R. S., Santini P., 2009, MNRAS, 397, 1776

Gargiulo I. D., et al., 2015, MNRAS, 446, 3820

Gavazzi G., Fumagalli M., Cucciati O., Boselli A., 2010, A\&A, 517, A73

Gisler G. R., 1976, A\&A, 51, 137

Guglielmo V., Poggianti B. M., Moretti A., Fritz J., Calvi R., Vulcani B., Fasano G., Paccagnella A., 2015, MNRAS, 450, 2749

Gunn J. E., Gott J. Richard I., 1972, ApJ, 176, 1

Haines C. P., La Barbera F., Mercurio A., Merluzzi P., Busarello G., 2006, ApJ, 647, L21

Haines C. P., et al., 2013, ApJ, 775, 126

Haines C. P., et al., 2015, ApJ, 806, 101

Heß S., Springel V., 2012, MNRAS, 426, 3112

Jáchym P., Palouš J., Köppen J., Combes F., 2007, A\&A, 472, 5

Jaffé Y. L., Smith R., Candlish G. N., Poggianti B. M., Sheen Y.-K., Verheijen M. A. W., 2015, MNRAS, 448, 1715

Jaffé Y. L., et al., 2018, MNRAS, 476, 4753

Jung S. L., Choi H., Wong O. I., Kimm T., Chung A., Yi S. K., 2018, ApJ, 865,156

Kauffmann G., et al., 2003, MNRAS, 341, 33

Kawata D., 2008, Astronomical Herald, 101, 214

Kovač K., et al., 2014, MNRAS, 438, 717

Kronberger T., Kapferer W., Ferrari C., Unterguggenberger S., Schindler S., 2008, A\&A, 481, 337

Lacey C., Cole S., 1993, MNRAS, 262, 627

Lagos C. D. P., Cora S. A., Padilla N. D., 2008, MNRAS, 388, 587

Lan T.-W., Ménard B., Mo H., 2016, MNRAS, 459, 3998

Larson R. B., Tinsley B. M., Caldwell C. N., 1980, ApJ, 237, 692

Lea S. M., De Young D. S., 1976, ApJ, 210, 647

Li Q., et al., 2020, MNRAS, 495, 2930

McCarthy I. G., Frenk C. S., Font A. S., Lacey C. G., Bower R. G., Mitchell N. L., Balogh M. L., Theuns T., 2008, MNRAS, 383, 593

McGee S. L., Balogh M. L., Bower R. G., Font A. S., McCarthy I. G., 2009, MNRAS, 400, 937

Merritt D., 1983, ApJ, 264, 24

Mostoghiu R., Knebe A., Cui W., Pearce F. R., Yepes G., Power C., Dave R., Arth A., 2019, MNRAS, 483, 3390

Muñoz Arancibia A. M., Navarrete F. P., Padilla N. D., Cora S. A., Gawiser E., Kurczynski P., Ruiz A. N., 2015, MNRAS, 446, 2291

Muzzin A., et al., 2012, ApJ, 746, 188

Muzzin A., et al., 2014, ApJ, 796, 65

Nantais J. B., et al., 2016, A\&A, 592, A161 
Nantais J. B., et al., 2017, MNRAS, 465, L104

Oman K. A., Hudson M. J., Behroozi P. S., 2013, MNRAS, 431, 2307

Orsi Á., Padilla N., Groves B., Cora S., Tecce T., Gargiulo I., Ruiz A., 2014, MNRAS, 443, 799

Pallero D., Gómez F. A., Padilla N. D., Torres-Flores S., Demarco R., Cerulo P., Olave-Rojas D., 2019, MNRAS, 488, 847

Pallero D., Gómez F. A., Padilla N. D., Bahé Y. M., Vega-Martínez C. A., Torres-Flores S., 2020, arXiv e-prints, p. arXiv:2012.08593

Peng Y.-j., et al., 2010, ApJ, 721, 193

Peng Y.-j., Lilly S. J., Renzini A., Carollo M., 2012, ApJ, 757, 4

Peng Y., Maiolino R., Cochrane R., 2015, Nature, 521, 192

Poggianti B. M., et al., 2016, AJ, 151, 78

Press W. H., Schechter P., 1974, ApJ, 187, 425

Quilis V., Moore B., Bower R., 2000, Science, 288, 1617

Rasmussen J., Ponman T. J., Verdes-Montenegro L., Yun M. S., Borthakur S., 2008, MNRAS, 388, 1245

Roberts I. D., Parker L. C., Brown T., Joshi G. D., Hlavacek-Larrondo J., Wadsley J., 2019, ApJ, 873, 42

Rodriguez Wimberly M. K., Cooper M. C., Fillingham S. P., Boylan-Kolchin M., Bullock J. S., Garrison-Kimmel S., 2019, MNRAS, 483, 4031

Roediger E., Brüggen M., 2006, MNRAS, 369, 567

Roediger E., Brüggen M., 2007, MNRAS, 380, 1399

Roediger E., Brüggen M., 2008, MNRAS, 388, 465

Roediger E., Hensler G., 2005, A\&A, 433, 875

Roediger E., Brüggen M., Hoeft M., 2006, MNRAS, 371, 609

Roediger E., Bruggen M., Owers M. S., Ebeling H., Sun M., 2014, MNRAS, 443, L114

Ruggiero R., Lima Neto G. B., 2017, MNRAS, 468, 4107

Ruiz A. N., et al., 2015, ApJ, 801, 139

Ruszkowski M., Brüggen M., Lee D., Shin M. S., 2014, ApJ, 784, 75

Somerville R. S., Davé R., 2015, ARA\&A, 53, 51

Springel V., Hernquist L., 2002, MNRAS, 333, 649

Springel V., White S. D. M., Tormen G., Kauffmann G., 2001, MNRAS, 328,726

Steinhauser D., Schindler S., Springel V., 2016, A\&A, 591, A51

Stevens A. R. H., Brown T., 2017, MNRAS, 471, 447

Takeda H., Nulsen P. E. J., Fabian A. C., 1984, MNRAS, 298, 261

Taylor E. N., et al., 2015, MNRAS, 446, 2144

Tecce T. E., Cora S. A., Tissera P. B., Abadi M. G., Lagos C. D. P., 2010, MNRAS, 408, 2008

Tecce T. E., Cora S. A., Tissera P. B., 2011, MNRAS, 416, 3170

Thomas D., Maraston C., Schawinski K., Sarzi M., Silk J., 2010, MNRAS, 404, 1775

Toniazzo T., Schindler S., 2001, MNRAS, 325, 509

Tonnesen S., Bryan G. L., 2008, ApJ, 684, L9

Tonnesen S., Bryan G. L., 2010, ApJ, 709, 1203

Tonnesen S., Bryan G. L., 2012, MNRAS, 422, 1609

Toyama K., Ikeuchi S., 1980, Progress of Theoretical Physics, 64, 831

Vijayaraghavan R., Ricker P. M., 2013, MNRAS, 435, 2713

Vijayaraghavan R., Ricker P. M., 2015, MNRAS, 449, 2312

Vollmer B., Cayatte V., Balkowski C., Duschl W. J., 2001, ApJ, 561, 708

Weinmann S. M., Kauffmann G., von der Linden A., De Lucia G., 2010, MNRAS, 406, 2249

Wetzel A. R., Tinker J. L., Conroy C., 2012, MNRAS, 424, 232

Wetzel A. R., Tinker J. L., Conroy C., van den Bosch F. C., 2013, MNRAS, 432, 336

Wetzel A. R., Tollerud E. J., Weisz D. R., 2015, ApJ, 808, L27

White S. D. M., Rees M. J., 1978, MNRAS, 183, 341

Xie L., De Lucia G., Hirschmann M., Fontanot F., 2020, MNRAS, 498, 4327

Zentner A. R., Berlind A. A., Bullock J. S., Kravtsov A. V., Wechsler R. H., 2005, ApJ, 624, 505

Zinger E., Dekel A., Kravtsov A. V., Nagai D., 2018, MNRAS, 475, 3654

van den Bosch F. C., Aquino D., Yang X., Mo H. J., Pasquali A., McIntosh D. H., Weinmann S. M., Kang X., 2008, MNRAS, 387, 79

van der Burg R. F. J., McGee S., Aussel H., Dahle H., Arnaud M., Pratt G. W., Muzzin A., 2018, A\&A, 618, A140

van der Burg R. F. J., et al., 2020, A\&A, 638, A112
This paper has been typeset from a $\mathrm{T}_{\mathrm{E}} \mathrm{X} / \mathrm{LAT} \mathrm{EX}$ file prepared by the author.

MNRAS 000, 1-15 (2021) 